\title{
Membrane ion transport in non-excitable tissues *
}

\author{
Keith Nehrke ${ }^{\S}$
}

Departments of Medicine (Nephrology Division) and Pharmacology and Physiology, University of Rochester Medical Center, Rochester NY 14642, USA

\section{Table of Contents}

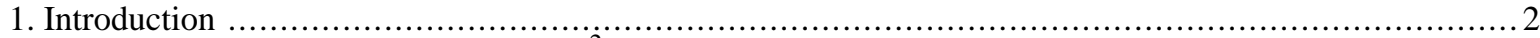

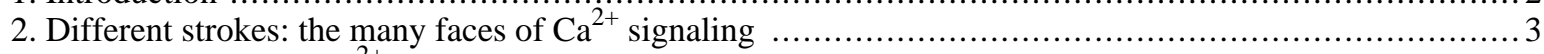

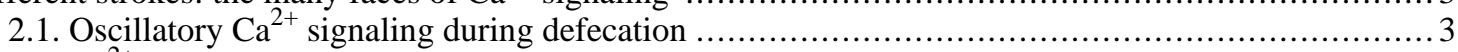

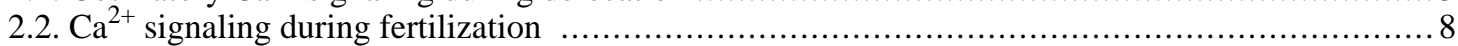

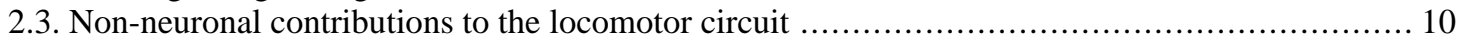

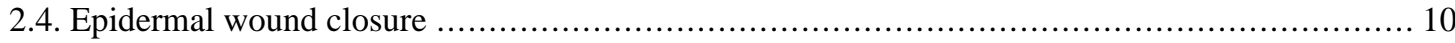

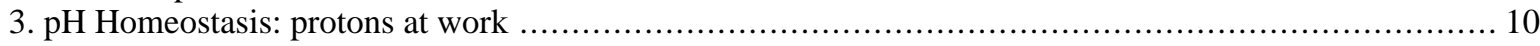

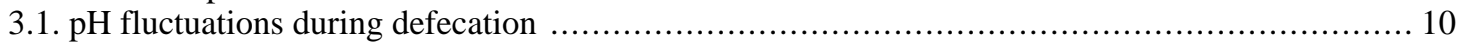

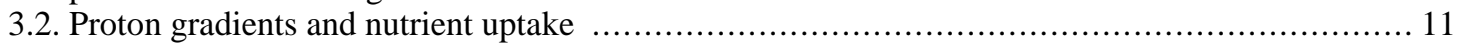

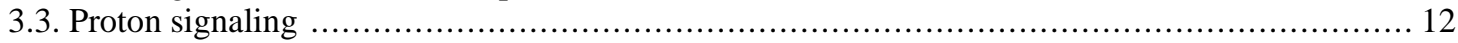

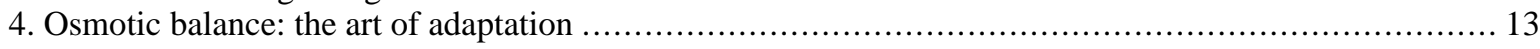

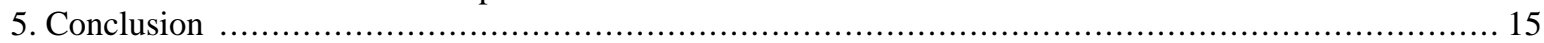

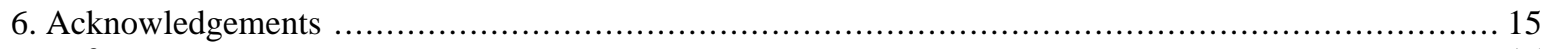

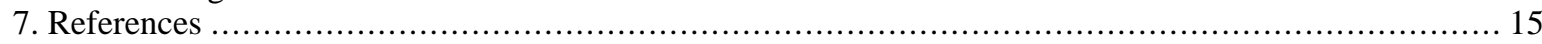

\begin{abstract}
The facilitated movement of ions across cell membranes can be characterized as occurring through active (ATP-dependent), secondary active (coupled), or passive transport processes. Each of these processes is mediated by a diverse group of membrane proteins. Over the past fifteen years, studies of membrane transport in $C$. elegans have benefited from the fact that worms are anatomically simple, easily and economically cultured, and genetically tractable. These experimental advantages have been instrumental in defining how membrane transport processes contribute to whole organism physiology. The focus of this review is to survey the recent advances in our understanding of membrane transport that have arisen from integrative physiological approaches in the nematode $C$. elegans.
\end{abstract}

\footnotetext{
*Edited by: Michel Labousse and Donald G. Moerman. Last Revised 6/30/2014. Published December 23, 2014. This chapter should be cited as: Nehrke K. Membrane ion transport in non-excitable tissues (December 23, 2014), WormBook, ed. The C. elegans Research Community, WormBook, doi/10.1895/wormbook.1.174.1, http://www.wormbook.org.

Copyright: (C) 2014 Keith Nehrke. This is an open-access article distributed under the terms of the Creative Commons Attribution License, which permits unrestricted use, distribution, and reproduction in any medium, provided the original author and source are credited.

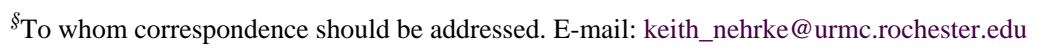




\section{Introduction}

The ability to form and maintain membrane electrochemical gradients is fundamental to life. Electrochemical gradients are created by the regulated distribution of ions by membrane transport proteins that function as molecular gatekeepers to move electrolytes and solutes across lipid bilayers. These proteins are generally classified into three categories: active, secondary active, and passive transporters. Active transporter function is coupled directly to ATP hydrolysis. One of the more well-recognized examples of this class is the $\mathrm{Na}^{+}-\mathrm{K}^{+}$ATPase, which establishes $\mathrm{Na}^{+}$ and $\mathrm{K}^{+}$electrochemical gradients across the cell membrane. The movement of $\mathrm{Na}^{+}$and $\mathrm{K}^{+}$down their electrochemical gradients can be harnessed by secondary active transporters to energize the movement of other solutes through coupled flux mechanisms against their electrochemical gradients. Unlike transporters that utilize energy directly or through secondary active processes, passive transporters such as ion channels provide a pathway for passive movement of solutes down their electrochemical gradients.

Early studies on membrane transporters measured their activity through biophysical techniques such as patch-clamp electrophysiology and radiolabeled substrate uptake without knowing the identity of the proteins responsible. These approaches were essential for characterizing substrate specificity and protein distribution, and resulted in the identification of a variety of pharmacologic reagents that saw widespread experimental use. Over the course of the "molecular revolution" many of the genes coding for the underlying transporter proteins were identified. This contributed to a reductionist approach aimed at understanding the function of individual gene products through recombinant expression strategies. Most recently, integrative approaches have focused on the processes through which individual molecular components work together to organize physiologic responses and ultimately to coordinate systemic outputs.

C. elegans is particularly well suited for defining the integrative biology of membrane transport processes. The worm genome is well defined and powerful forward and reverse genetic tools greatly facilitate the characterization of gene function and the identification of genetic pathways. Development has been well-characterized in C. elegans and the animal is anatomically simple, with a limited number of cells of invariant lineage comprising each organ system. In addition, worms exhibit a number of readily observable behaviors that are controlled by membrane transport processes, which provide robust phenotypes to measure in genetic and functional genomic screens.

The apparent simplicity of $C$. elegans belies a surprising level of conserved physiological sophistication. At their most basic level, membrane ion transporters exhibit a commonality of functions across phyla. This is underscored by the observation that transporter gene families are generally conserved between worms and mammals. While this might be expected for fundamental transporters such as the $\mathrm{Na}^{+}-\mathrm{K}^{+}$ATPase, it is also true for more specialized families of transporters. For example, there are nine $\mathrm{Na}^{+} / \mathrm{H}^{+}$exchanger genes in both humans and C. elegans. Although direct orthology has yet to be established for many of these, both human and C. elegans genomes code for paralogs with cell specific expression patterns and others with more global expression. The diversity of transporters in worms, as in mammals, allows them to function in individual tissues, in integrated physiologic processes that require the interaction of multiple organs, and to respond adaptively to environmental pressures.

Despite its many advantages, there are also some disadvantages that must be recognized when choosing to utilize the worm model. Foremost is the difficulty in obtaining access to individual cells in the organism due to what is effectively a pressurized cuticle. This has made traditional electrophysiological approaches difficult, though not impossible (Goodman et al., 1998; Richmond et al., 1999; Goodman et al., 2012). In addition, there are no $C$. elegans cell lines available for physiological and molecular studies. Techniques do exist, however, for generating dispersed primary embryonic cells of varying types that are accessible for electrophysiological and imaging approaches (Christensen et al., 2002; Strange et al., 2007; Culture of embryonic C. elegans cells for electrophysiological and pharmacological analyses). To some extent, these disadvantages have been circumvented by optogenetic approaches and the use of genetically encoded biosensors (for review, see Akerboom et al., 2013). These next generation technologies combined with the optical transparency and small size of $C$. elegans allow single cell measurements of electrolyte flux in live animals to be combined with optical control of membrane potential and intracellular signaling. Given the existing repertoire of genetic, reverse genetic, and transgenic reagents, these approaches firmly cement $C$. elegans as a robust model for studying the role of individual membrane transport processes in systems physiology. 


\section{Different strokes: the many faces of $\mathrm{Ca}^{2+}$ signaling}

$\mathrm{Ca}^{2+}$ is an ubiquitous second messenger whose function as a signaling molecule is specified by spatial and temporal constraints. In C. elegans, forward and reverse genetic approaches have identified physiologic processes that are linked to $\mathrm{Ca}^{2+}$ signaling, and the transparency of the worm has facilitated in vivo approaches using fluorescent Genetically Encoded $\mathrm{Ca}^{2+}$ Indicator (GECI) proteins to determine how $\mathrm{Ca}^{2+}$ flux is constrained. This section will focus on non-neuronal physiological processes where $\mathrm{Ca}^{2+}$ signaling is fundamental, exploring mechanisms for $\mathrm{Ca}^{2+}$ signal initiation, plasma membrane $\mathrm{Ca}^{2+}$ entry, and $\mathrm{Ca}^{2+}$ movement between cells.

Both defecation and fertilization are ultradian rhythmic behaviors that are timed by $\mathrm{Ca}^{2+}$ signaling. Unlike circadian rhythms which occur daily, their periods are on the order of minutes in well-fed animals. These short periods and stereotypical execution are key experimental advantages that have facilitated integrative molecular understanding of the underlying physiological processes. An additional advantage is that both defecation and fertilization are relatively simple behaviors that require the action of just several cells, with $\mathrm{Ca}^{2+}$ signaling required to maintain behavioral fidelity through both cell autonomous and non-autonomous mechanisms.

\subsection{Oscillatory $\mathrm{Ca}^{2+}$ signaling during defecation}

The defecation motor program (DMP) occurs with a period of $\sim 50$ s (Thomas, 1990) and consists of three stereotypical, visible outputs termed the posterior body contraction (pBoc), anterior body contraction (aBoc) and expulsion (Figure 1; Movie 1) (For review see Branicky et al., 2006). When worms are well-fed, the DMP occurs with little variation (Thomas, 1990). This basal rhythmicity, coupled with an ability to be entrained by external stimuli and insensitivity to temperature (Liu et al., 1994), matches the requirements for a "biological clock". Seminal work from the Jorgenson laboratory showed that mutations in itr-1, which codes for the sole worm inositol 1, 4, 5-trisphosphate receptor (IP3R), slow down or eliminate the cycle (Dal Santo et al., 1999). The IP3R is an endoplasmic reticulum (ER) $\mathrm{Ca}^{2+}$ release channel, and overexpression of the IP3R specifically in the intestine caused the cycle to speed up (Dal Santo et al., 1999). This observation was consistent with the finding that DMP rhythmicity was controlled independent of specific neuronal input. Based upon these data, cell-autonomous IP3 dependent $\mathrm{Ca}^{2+}$ signaling was proposed to be the molecular pacemaker for defecation. 


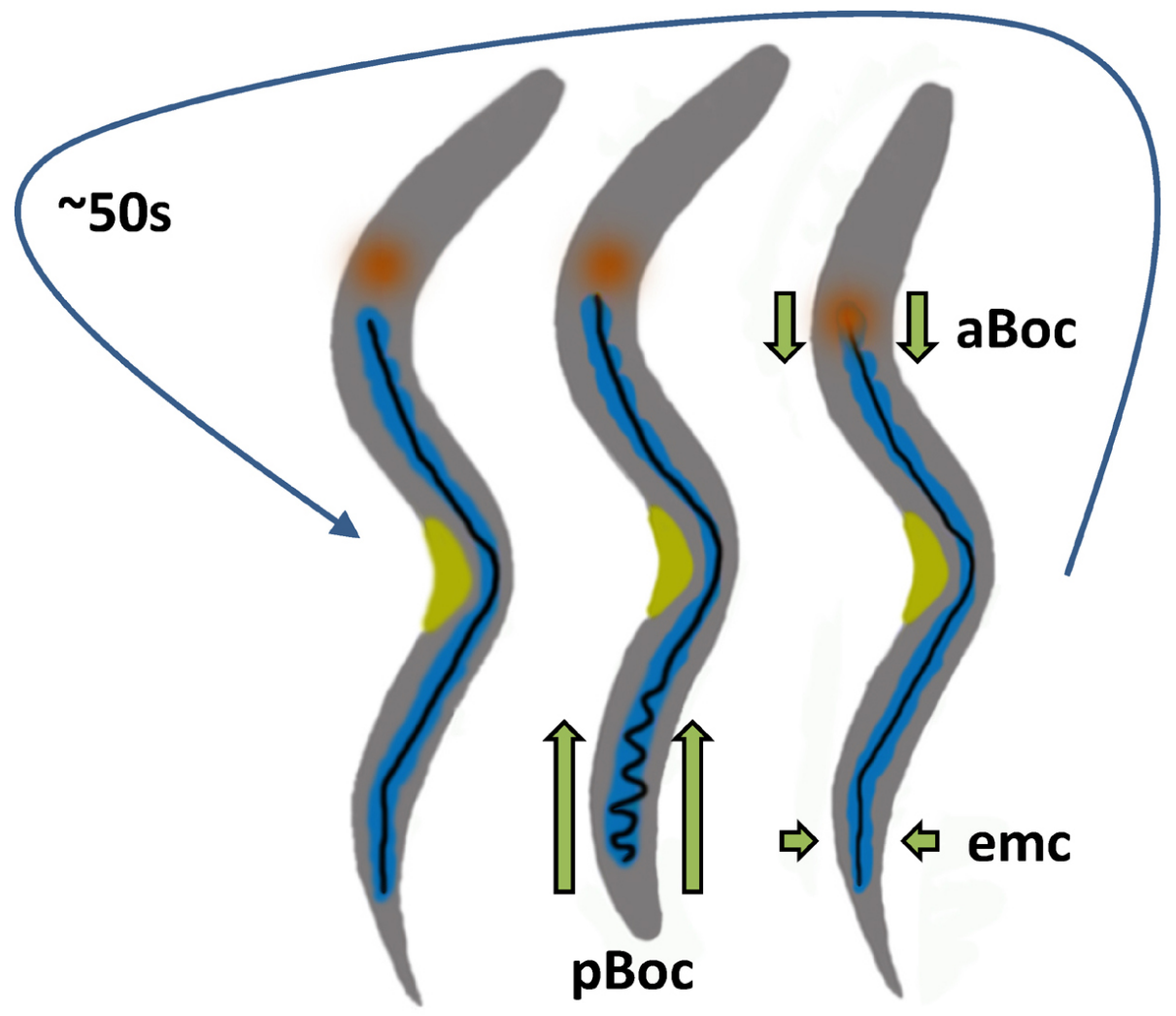

Figure 1. Schematic diagram of the defecation motor program (DMP). Every $\sim 50 \mathrm{~s}$, three independent sets of muscle contractions are carried out in a stereotypical sequence. The first step consists of $\mathrm{pBoc}$, a simultaneous contraction of the posterior body wall muscles on both sides of the worm. This step compresses the lumen in the posterior of the worm and moves the luminal contents forward. The second step, aBoc, occurs several seconds later and consists of a contraction of the anterior body wall muscles. This causes the terminal bulb of the pharynx to plunge into the anterior-most, bowl-like region of the intestinal lumen and results in the luminal contents moving backwards. Expulsion is then carried out by four coordinated enteric muscle contractions (emc; not shown in detail). Blue, intestine; orange, pharynx; black; intestinal lumen; yellow, vulva.

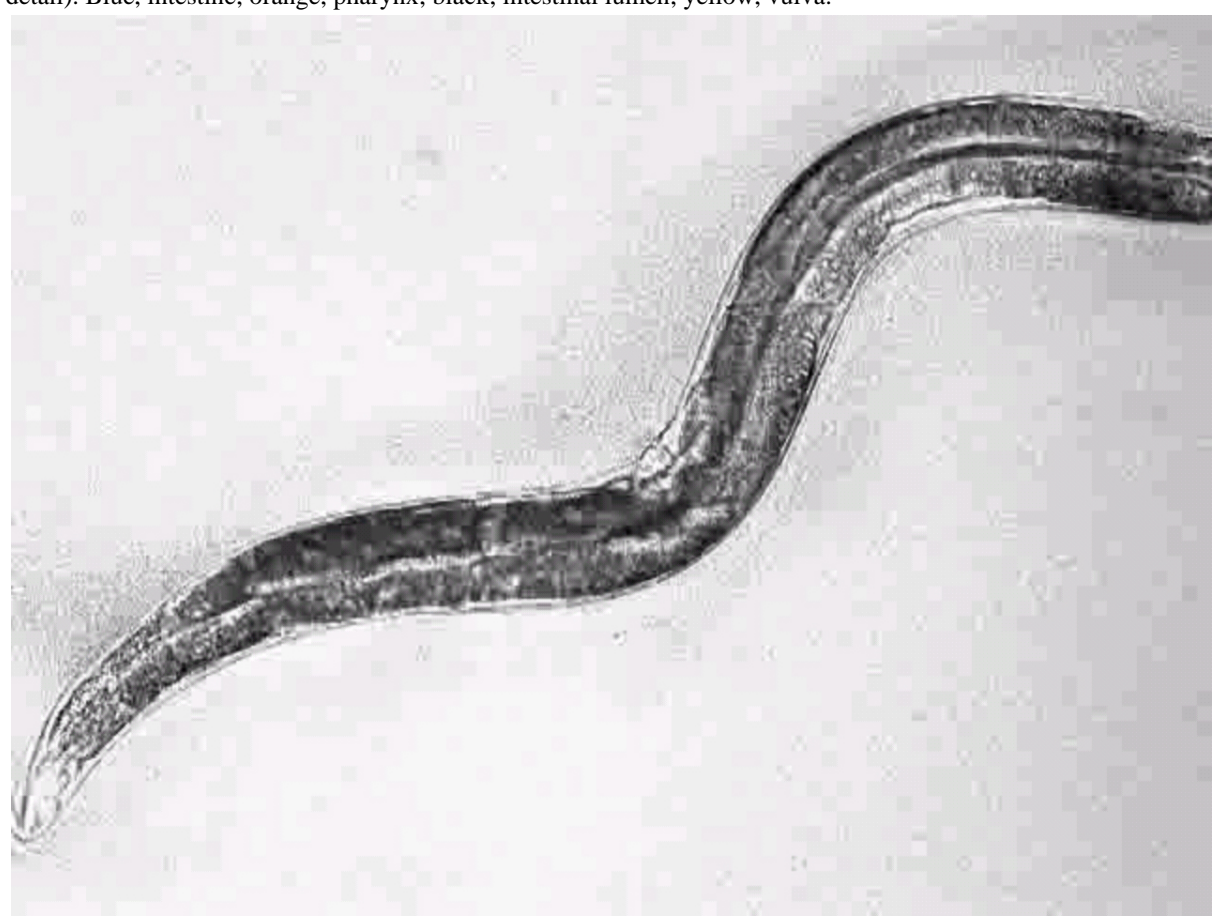

Movie 1. Wildtype worms executing the defecation motor program. Captured using transmitted light. 
Subsequently, it has been discovered that a wave of $\mathrm{Ca}^{2+}$ initiates in the posterior intestinal cells and propagates forward through the intestine concomitant with execution of the DMP (Figure 2; Movie 2); several parallel approaches that utilized isolated intact intestinal preparations loaded with $\mathrm{Ca}^{2+}$ sensitive dyes or live worms expressing fluorescent GECIs combined with dynamic imaging techniques have corroborated this finding (Espelt et al., 2005; Norman et al., 2005; Teramoto et al., 2006; Peters et al., 2007; Nehrke et al., 2008). INX-16, an innexin gap junction protein, facilitates the propagation of the $\mathrm{Ca}^{2+}$ wave between individual cells of the intestine (Peters et al., 2007; Coburn et al., 2013). $\mathrm{Ca}^{2+}$ wave dynamics couple the sequential motor steps of the DMP (Teramoto et al., 2006), and $\mathrm{Ca}^{2+}$ signaling in the anterior intestinal cells has been suggested to regulate aBoc timing independent of the basal pacemaker (Nehrke et al., 2008; Wang et al., 2013). Hence, $\mathrm{Ca}^{2+}$ oscillations in the posterior intestinal int9 cell are the pacemaker that defines the DMP period and the speed of $\mathrm{Ca}^{2+}$ wave propagation is likely the defining factor that controls temporal coupling between the first and second motor steps.

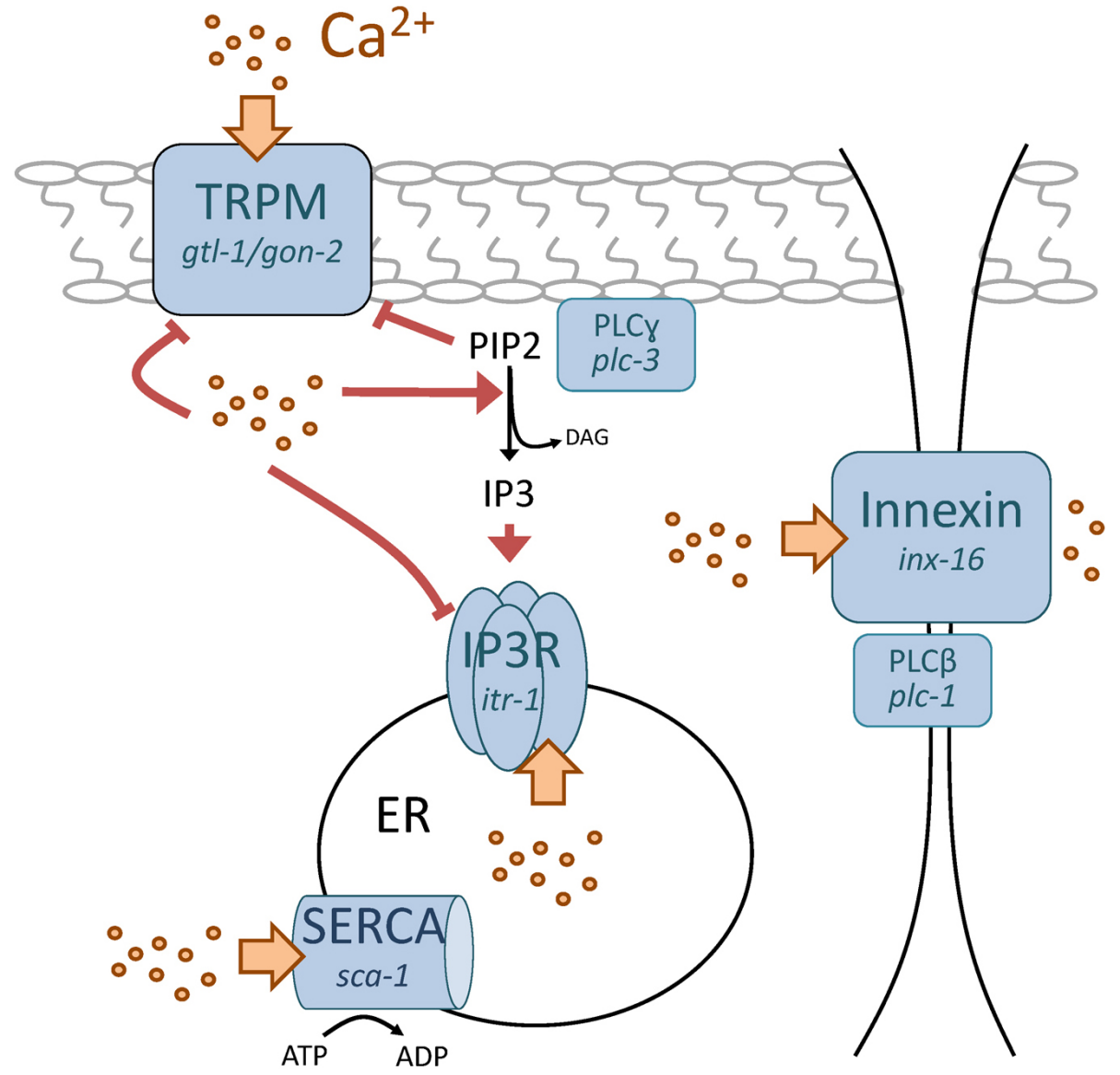

Figure 2. Schematic diagram indicating $\mathrm{Ca}^{2+}$ transporters that time defecation. Proteins are shown in blue. $\mathrm{Ca}^{2+}$ is represented by yellow dots, with movement through the transporters denoted by yellow arrows. Positive and negative regulation by $\mathrm{Ca}^{2+}$ is shown in red, with lipid signaling molecules in black. Nematode genes are in italics under the more widely-used protein names. In brief, oscillations in cytoplasmic $\mathrm{Ca}^{2+}$ are thought to occur through sequential PLC $\gamma$ mediated stimulation of the IP3R concurrent with de-suppression of TRPM, followed by ER store $\mathrm{Ca}^{2+}$ release and $\mathrm{Ca}^{2+}$ feedback regulation of the three proteins involved. $\mathrm{Ca}^{2+}$ moves through cells via an insect gap junction innexin, which contributes to $\mathrm{Ca}^{2+}$ wave propagation. PLC $\beta$ has an undefined role in wave propagation that is independent of the IP3R, but may relate to its presence at the gap junction. Store repletion by the SERCA pump restores $\mathrm{Ca}^{2+}$ to baseline levels. Inositol 1, 4, 5-trisphosphate (IP3), IP3 Receptor (IP3R), Transient Receptor Potential Melastatin (TRPM), phospholipase C (PLC), sarco-endoplasmic reticular $\mathrm{Ca}^{2+}$ ATPase (SERCA), phosphatidylinositol 4,5-bisphosphate (PIP2), diacylglycerol (DAG), endoplasmic reticulum (ER). 


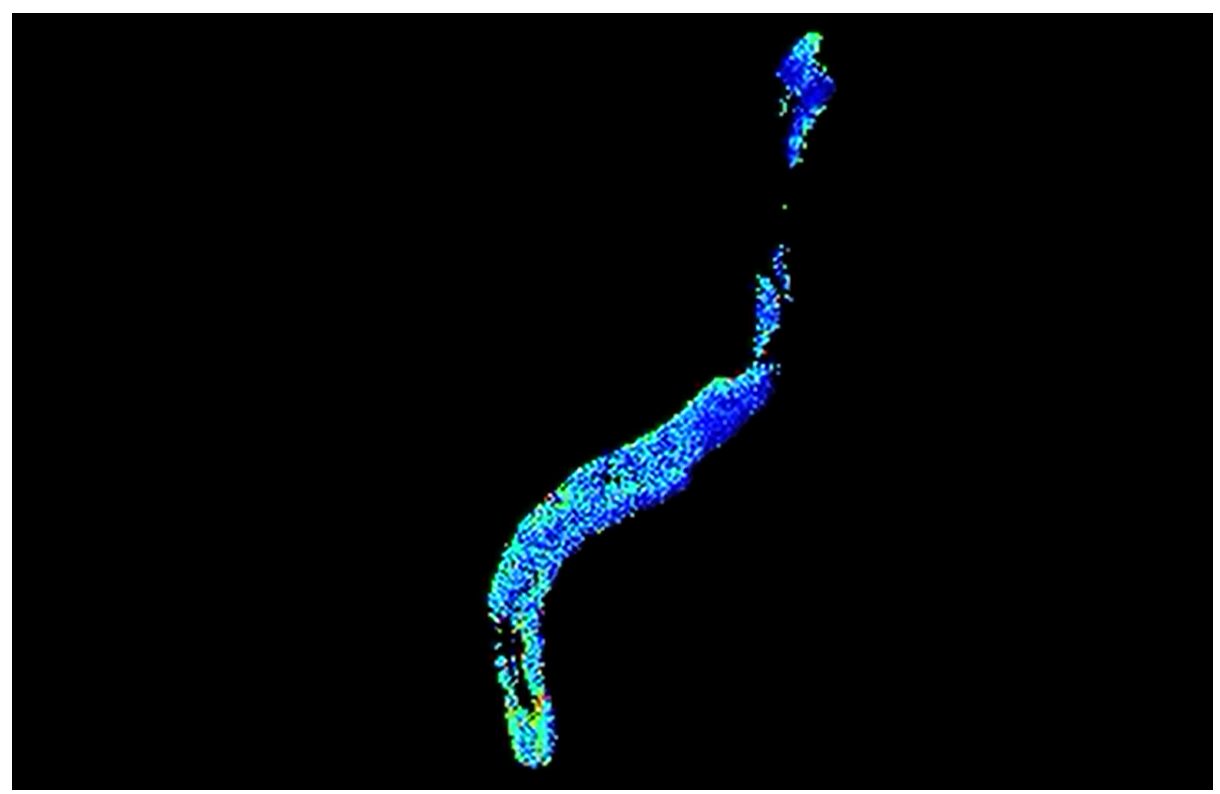

Movie 2. Worms expressing the fluorescent $\mathrm{Ca}^{2+}$ biosensor D3cpv in the intestine executing the defecation motor program. FRET analysis was used to create a pseudocolor ratio map from time series of single excitation dual emission fluorescent images obtained from unrestrained worm moving normally on agar plates seeded with bacteria. Blue, low $\mathrm{Ca}^{2+} ;$ red, high $\mathrm{Ca}^{2+}$.

The supremacy of the posterior-most intestinal cell int 9 as the pacemaker cell was recently shown to require an miRNA that regulates fatty acid biosynthesis (Kemp et al., 2012). mir-786 is expressed in int9, and a defect in timing and execution of the DMP was shown to result from aberrant $\mathrm{Ca}^{2+}$ wave initiation in mir-240/786 mutant worms (Kemp et al., 2012). This defect resulted from a loss of mir-786 suppression of the fatty acid elongase elo-2, and palmitate supplementation was sufficient to suppress the long arrhythmic defecation cycles in the mutants (Kemp et al., 2012). The current model suggests that the fatty acid composition in int 9 sensitizes this cell such that its oscillatory period is slightly faster than other cells in the intestine, and perhaps the $\mathrm{Ca}^{2+}$ signal is stronger, though this has not been tested directly. Since $\mathrm{Ca}^{2+}$ regulates the IP3R through feedback inhibition, normal $\mathrm{Ca}^{2+}$ wave propagation through the intestine would be expected to suppress independent oscillations in $\mathrm{Ca}^{2+}$ that might occur in other cells. Since mir-786 acts upstream of the IP3R, which is the central molecular pacemaker for defecation, it is possible that fatty acids directly influence the IP3R's activity (Kemp et al., 2012). Together, these results suggest that mir-786 is an amplifier of $\mathrm{Ca}^{2+}$ signaling. Since mir-786 is expressed from a gene cluster including mir-240 and both of these genes as well as their positioning are conserved in mammals (where they are termed, "miR-193b-365"), it will be interesting to see whether the same holds true in higher level eukaryotes.

There are two potential types of ion transport mechanisms that could facilitate plasma membrane $\mathrm{Ca}^{2+}$ entry to support intestinal $\mathrm{Ca}^{2+}$ wave propagation and electrophysiological approaches using a primary culture system (Christensen et al., 2002) for functional analysis of $C$. elegans intestinal epithelial cells have revealed that both of these exist: store-independent and store-operated $\mathrm{Ca}^{2+}$ entry (SOCE) pathways (Estevez et al., 2003). "Store-operated" refers to the regulatory process through which plasma membrane $\mathrm{Ca}^{2+}$ entry is coupled to $\mathrm{ER} \mathrm{Ca}^{2+}$ depletion. IP3R and ER $\mathrm{Ca}^{2+}$ stores are central to the defecation pacemaker (Dal Santo et al., 1999). IP3R opening results in $\mathrm{Ca}^{2+}$ depletion from the ER, which can lead to the activation of SOCE pathways into the cell. Over the past several years, the molecular identities of the channels responsible for canonical SOCE and their mechanism of regulation have been discovered. Stim1 is an EF-hand $\mathrm{Ca}^{2+}$ binding protein that resides in the ER and senses store depletion (Roos et al., 2005; Zhang et al., 2005) while Orail is an essential pore-forming subunit whose opening is regulated by Stim1 (Prakriya et al., 2006; Yeromin et al., 2006). The $C$. elegans stim-1 and orai-1 gene products are expressed in tissues where regular $\mathrm{Ca}^{2+}$ oscillations occur, such as the pharynx, spermatheca, and intestinal cells (Strange et al., 2007). Patch-clamp electrophysiological recordings of co-expressed stim-1 and orai-1 cDNAs recapitulate the biophysical transport properties of native SOCE currents (Lorin-Nebel et al., 2007). However, neither orai-1 nor stim-1 appears to play a role in defecation. Their loss compromises the ability to maintain homeostasis under conditions of ER stress, suggesting that they are functional, but has no effect on the DMP behavior or oscillatory $\mathrm{Ca}^{2+}$ signaling in the intestine (Yan et al., 2006). So how do intestinal epithelial cells in worms take up $\mathrm{Ca}^{2+}$ during the DMP? 
An alternative to Orail/Stim1 was provided by the observation that a store-independent outward rectifying $\mathrm{Ca}^{2+}$ conductance termed $\mathrm{I}_{\mathrm{ORCa}}$ found in primary isolated embryonic intestinal cells oscillates under conditions of low $\mathrm{Ca}^{2+}$ buffering (Estevez et al., 2005). The biophysical properties of $\mathrm{I}_{\mathrm{ORCa}}$ resembled those of Transient Receptor Potential Melastatin (TRPM) channels, a class of a superfamily of non-selective cation channels that are conserved from worms to humans. Two TRPM homologs, gon-2 and gtl-1, are expressed in the nematode intestine. These two channels have nearly identical biophysical transport properties, are independently required for normal I to maintain behavioral rhythmicity during defecation by facilitating the influx of $\mathrm{Ca}^{2+}$ to the cell (Kwan et al., 2008 ; Xing et al., 2008). Hence, $\mathrm{Ca}^{2+}$ oscillations in the worm intestine appear to be supported by non-canonical store-independent $\mathrm{Ca}^{2+}$ entry pathways.

However, despite these findings, the specific role of GON-2 and GTL-1 in regulating defecation has been debated and their loss-of-function defecation phenotypes have been suggested to result indirectly from defects in magnesium absorption (Teramoto et al., 2005). Supporting this argument is the fact that the orthologous mammalian TRPM6 and TRPM7 channel-kinases govern intestinal magnesium uptake (Quamme, 2008). However, worm TRPM channels lack the atypical alpha kinase domain (AAKD) found on both TRPM6 and TRPM7 (Runnels, 2011). AAKDs are widespread amongst vertebrates and form a distinct family with specific substrate preferences. Other members of this family include erukaryotic elongation factor-2 kinase and myosin heavy chain kinase. The extent to which this domain might influence magnesium uptake is currently unknown.

An alternative theory was proposed where coupling of IP3R activity with plasma membrane $\mathrm{Ca}^{2+}$ influx through TRPM channels occurred through co-regulation by phosphatidylinositol 4,5-bisphosphate (PIP2) in conjunction with $\mathrm{Ca}^{2+}$ itself (Xing et al., 2010). Membrane phosphoinositides play a fundamental role in regulating the activity of many plasma membrane transporters (Huang, 2007). For example, the IP3R is activated by phospholipase C (PLC)-mediated hydrolysis of PIP2 into diacylglycerol and IP3. And while many ion channel classes bind to and are activated by PIP2, the TRPM channels GTL-1 and GON-2 are inhibited by PIP2 (Xing et al., 2010). Although this suggests that they may be functional outliers in terms of modulation by PIP2, it does allow for an interesting hypothesis: PIP2 hydrolysis following PLC activation could stimulate both IP3R and TRPM channel activities (model shown in Figure 2). In conjunction, $\mathrm{Ca}^{2+}$ is a positive regulator of PLC activity, a negative regulator of TRPM channel activity, and a biphasic regulator of IP3R: low concentrations of cytoplasmic $\mathrm{Ca}^{2+}$ activate and conversely high concentrations inhibit further $\mathrm{Ca}^{2+}$ release from the ER. As Ca ${ }^{2+}$ levels increase in the cytoplasm, IP3R and TRPM activity would be predicted to be reduced by feedback inhibition (Estevez et al., 2005), which would allow intracellular stores to refill and cytoplasmic $\mathrm{Ca}^{2+}$ to return to baseline levels. As this occurs, TRPM channel feedback inhibition would be relieved, allowing small amounts of $\mathrm{Ca}^{2+}$ to enter the cell, subsequently activating PLC. Increasing PLC activity would then lower PIP2 levels and desuppress TRPM channel activity, resulting in a self-sustaining oscillatory system in the absence of stimulation by extracellular ligands.

C. elegans contain six PLC isoforms, and consistent with the above model PLC $\gamma$ (plc-3) regulates the behavioral rhythmicity of the DMP upstream of the IP3R (Espelt et al., 2005). Hence, PLC-3 activity likely plays the role shown in Figure 2. PLC $\beta$ (plc-1/egl-8) activity also contributes to defecation rhythmicity, but unlike PLC $\gamma$ does not functionally interact with the IP3R (Espelt et al., 2005). PLC $\beta$ has been localized to intestinal cell junctions (Miller et al., 1999) and its loss results in abnormal $\mathrm{Ca}^{2+}$ wave propagation (Nehrke et al., 2008), much like that observed following the loss of the gap junction protein innexin INX-16 (Peters et al., 2007) or mir-786 (Kemp et al., 2012) This suggests that PLC-1 regulates gap junction movement of $\mathrm{Ca}^{2+}$ and is consistent with the idea that wave-driven elevations in $\mathrm{Ca}^{2+}$ suffice to suppress cell autonomous $\mathrm{Ca}^{2+}$ oscillations in cells anterior to int9.

Further evidence for the role of PIP2-IP3 metabolism being central to defecation pacemaking comes from a mutation in the guanidine nucleotide exchange factor (GEF) VAV-1 that alters several rhythmic behaviors, including defecation (Norman et al., 2005). The small GTPases ced-10, mig-2, and rho-1 act redundantly in a pathway with $v a v-1$ to modulate IP3 levels and may directly regulate $p p k-1$ (PIP5K) activity. GEFs generally function to integrate environmental signals transduced through cell surface receptor activation, but it is as-of-yet unclear what these signals are in worms.

Following defecation, ER $\mathrm{Ca}^{2+}$ stores are replenished through the endoplasmic reticular $\mathrm{Ca}^{2+} \mathrm{ATPase}^{2+}$ (SERCA) SCA-1. Though a loss-of-function mutation in sca-1 is lethal, mild intestine-restricted RNAi has been used to probe its influence on $\mathrm{Ca}^{2+}$ oscillations during defecation. Surprisingly, mild intestinal knockdown resulted in uncoordinated pBoc and aBoc (Nehrke et al., 2008). Previously, it had been shown that inhibiting Ca ${ }^{2+}$ wave propagation through the intestine also dissociated pBoc from aBoc (Teramoto et al., 2006). Although pBoc does not require neural input, AVL and DVB neurons are required for aBoc and exp. More recently, the execution of aBoc 
has been attributed to secretion of the neuropeptide-like protein NLP-40 from the intestine to these neurons (Wang et al., 2013). Hence, the $s c a-1(R N A i)$ phenotype is consistent with a role for $\mathrm{Ca}^{2+}$ dynamics in the anterior intestine controlling neuropeptide release and intestine-to-neuron signaling.

\section{2. $\mathrm{Ca}^{2+}$ signaling during fertilization}

Worms typically have 200-300 progeny, with oocytes undergoing fertilization every 23 minutes. Immature oocytes are arrested in the proximal gonad in meiotic prophase I, surrounded by smooth muscle-like myoepithelial sheath cells. Meiosis is resumed just prior to fertilization in a process called meiotic maturation (Control of oocyte meiotic maturation and fertilization). Prior to meiotic maturation, sheath cells contract weakly and intermittently at a basal rate of 7-8 contractions/minute. However, meiotic maturation of the oocyte increases the force and rate of sheath contractions dramatically. The increased contraction rate is coupled to spermatheca dilation, which allows the mature oocyte to be inserted into the spermatheca for fertilization. This cycle is then repeated, and $\mathrm{Ca}^{2+} \mathrm{serves}$ to coordinate somatic and germline cell processes that need to occur in sequence for fertilization to occur properly (for review, see Singaravelu et al., 2013).

A key event that times fertilization is the secretion of major sperm protein (MSP), which stimulates parallel responses in both oocytes and the sheath cell. MSP stimulates meiotic maturation in the proximal most oocyte (Miller et al., 2001) by functioning as an ephrin-signaling antagonist and by counteracting inhibitory inputs from the somatic gonadal sheath cells (Govindan et al., 2006). Genetic analyses have suggested a complex regulatory cascade where the IP3R ITR-1 represses meiotic maturation downstream of the ephrin receptor VAB-1, and the Ca ${ }^{2+}$ channel NMDA receptor NMR-1 acts in a parallel pathway to regulate the maturation effector $\mathrm{Ca}^{2+}$ calmodulin $^{2}$ kinase type II UNC-43 (Corrigan et al., 2005). Hence, $\mathrm{Ca}^{2+}$ signaling may be important for keeping oocytes arrested in prophase I and for meiotic maturation as well, acting through different outputs (Figure 3). 


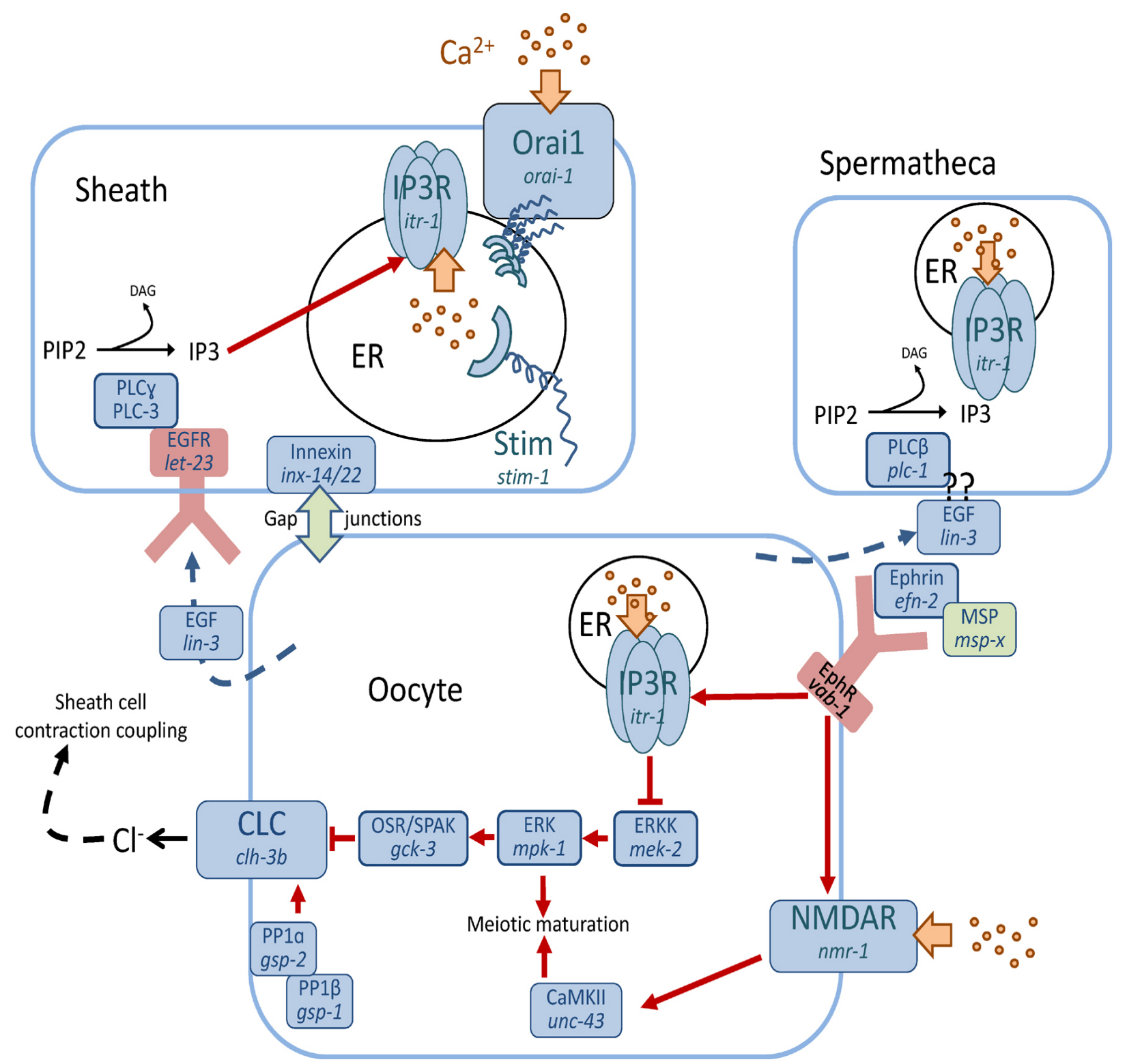

Figure 3. Schematic diagram indicating $\mathrm{Ca}^{2+}$ transport and signaling pathways that regulate fertilization. $\mathrm{Ca}^{2+}$ is represented by yellow dots, with its transport indicated by a yellow block arrow. Red arrows denote regulatory pathways. Several different cell types are important for fertilization, including sheath cells, spermatheca and the oocyte. The role of each of these cells has to be precisely coordinated in order for effective fertilization to occur. Hence, communication between cells is essential. $\mathrm{Ca}^{2+}$ signaling pathways have been shown to operate through discrete mechanisms in each cell to coordinate fertilization and assure the proper sequence of events. Inositol 1, 4, 5-trisphosphate (IP3), IP3 Receptor (IP3R), Voltage activated chloride channel (CLC), Oxidative stress responsive kinase 1/SPS1-related proline/alanine-rich kinase (OSR/SPAK), Protein phosphatase (PP), Ca ${ }^{2+}$ release-activated $\mathrm{Ca}^{2+}$ modulator 1 (Orai1), Stromal interaction molecule (Stim), Extracellular signal related kinase (ERK), ERK kinase (ERKK), $\mathrm{Ca}^{2+}$-calmodulin dependent kinase type II (CaMKII), Epidermal grown factor (EGF), EGF receptor, (EGFR), Ephrin receptor (EphR), Major sperm protein (MSP), N-methyl-D-aspartate receptor (NMDAR), phospholipase C (PLC), phosphatidylinositol 4,5-bisphosphate (PIP2), diacylglycerol (DAG), endoplasmic reticulum (ER).

During meiotic maturation, oocytes secrete the EGF ligand LIN-3 which binds to its receptor LET-23 on both the spermatheca and the sheath cell surface (Clandinin et al., 1998; Yin et al., 2004). This triggers PLC $\gamma$ (PLC-3) activity in the sheath cell to produce IP3. Levels of IP3 can be reduced by IP3 kinase LFE-2 or type I polyphosphate 5-phosphatase IPP-5, and loss-of-function mutations in lfe-2 or ipp-5 increase IP3 concentrations, suppressing the ovulation defect caused by lin-3 mutations; this holds true for a gain-of-function mutation in itr-1, the IP3 receptor, as well (Clandinin et al., 1998; Bui et al., 2002; Yin et al., 2004). A mutant allele of ipp-5 also exhibits an unusual ovulation phenotype in which the spermatheca hyperextends, thereby ovulating two oocytes per cycle (Bui et al., 2002), confirming that the IP3R is central to this cell's function in fertilization. The final step in fertilization occurs 
when the oocytes exit the spermatheca, and signaling through this pathway apparently requires PLCE (PLC-1) rather than PLC-3 (Kariya et al., 2004). Coordinated constriction of the myoepithelial tube following fertilization is mediated by mechanical stretch via the scaffolding protein filamin (FLN-1), which times PLC-1 activity, resulting in a distinctive series of IP3-dependent $\mathrm{Ca}^{2+}$ oscillations propagated through the tissue by gap junctions (Kovacevic et al., 2013). Together, these results suggest that $\mathrm{Ca}^{2+}$ signaling downstream of IP3R activation is sufficient to time and coordinate fertilization events.

Finally, unlike $\mathrm{Ca}^{2+}$ signaling during defecation, which acts through a non-canonical TRPM dependent pathway (Kwan et al., 2008; Xing et al., 2008), the increased rate and force of gonadal sheath cell contractions caused by LIN-3 are dependent upon both stim-1 and orai-1 (Lorin-Nebel et al., 2007). In a now-routine example of mechanistic convergence between worms and mammals, both Stim1 and Orai have recently been shown to function in oocyte meiotic maturation in mice (Cheon et al., 2013).

\subsection{Non-neuronal contributions to the locomotor circuit}

Systemic electrolyte homeostasis can influence signaling events that require coordinated action between multiple cells types, likely by altering the environment through which intercellular signaling processes must occur. For example, the TRPM channel GTL-2 was identified through an unbiased genetic screen for suppressors of the acr-2 gain-of-function $(g f)$ epilepsy-like phenotype, where gtl-2 loss-of-function restored excitation-inhibition imbalance in an $a c r-2(g f)$ strain (Stawicki et al., 2011). Cell-specific rescue analysis suggested the GTL-2 functions in the hypodermis or excretory cell (Stawicki et al., 2011). Like all TRP channels, GTL-2 is a non-selective cation channel capable of passing a variety of positively charged electrolytes, and it has been linked to magnesium excretion in the excretory cell (Teramoto et al., 2010). However, studies using relatively specific cation chelators suggest that its ability to suppress the $a c r-2(g f)$ phenotype may be related more to zinc homeostasis than to that of either $\mathrm{Ca}^{2+}$ or magnesium (Stawicki et al., 2011), exemplifying the complexity inherent to an integrated system. It is possible that the relative non-selectivity of TRP channels (such as GTL-2, or GON-2 and GTL-1) for different cations may diversify their effect on physiologic behaviors, complicating the interpretation of genotype-phenotype approaches.

\subsection{Epidermal wound closure}

Lesions to the worm's "skin" are repaired in part through a $\mathrm{G} \alpha-\mathrm{Ca}^{2+}$ signaling pathway required for actin-dependent wound closure (Xu et al., 2011). Cytoplasmic $\mathrm{Ca}^{2+}$ influx in hypodermal cells following wounding also occurs through GTL-2 (Xu et al., 2011), consistent with a role for this channel in passing a variety of cations. Wounds to a Drosophila embryo also result in a nearly instantaneous $\mathrm{Ca}^{2+}$ flash, suggesting that this mechanism represents one of the earliest signals in the wound response (Razzell et al., 2013). Interestingly, reactive oxygen species (ROS) signaling has been implicated in wound responses in both plants and animals (Suzuki et al., 2012) and several of the mammalian TRPM channels are either regulated by ROS (TRPM2) or have been implicated in survival responses to oxidative stress (TRPM7) (Sumoza-Toledo et al., 2011; Chen et al., 2012).

\section{3. pH Homeostasis: protons at work}

Cellular $\mathrm{pH}$ homeostasis is well protected by a variety of buffering and acid-base transport mechanisms. Both protons and hydroxyl ions can be transported across cell membranes much like other electrolytes, and the major biological buffer in the cell is bicarbonate which itself is a substrate for membrane transport. Gene families involved in acid-base transport and cell buffering, including sodium-proton exchangers, anion bicarbonate transporters and carbonic anhydrases, are well conserved between worms and mammals (Nehrke et al., 2002; Sherman et al., 2005; Bretscher et al., 2011; Fasseas et al., 2011; Sherman et al., 2012).

\section{1. $\mathrm{pH}$ fluctuations during defecation}

Proton transport processes and $\mathrm{pH}$ homeostasis are integral parts of the worm defecation cycle (Figure 4). As in regions of the mammalian gastrointestinal tract such as the stomach, the lumen of the worm's intestine is relatively acidic with a resting $\mathrm{pH} \approx 4.1$ (Pfeiffer et al., 2008). Recent work has shown that protons or proton equivalents move using an unidentified transport mechanism from the intestinal lumen into the cytoplasm of the cells (which are $\mathrm{pH} 7.5$ ) in response to $\mathrm{Ca}^{2+}$ oscillations during defecation (Pfeiffer et al., 2008). As a consequence, the proton gradient between the lumen and the cytoplasm dissipates. The cytoplasm becomes quite acidic $(\mathrm{pH} \approx 7)$ for a short period of time and the lumen becomes more alkaline ( $\mathrm{pH}>6)$. Only after expulsion has occurred are the protons transported back into the lumen. The return of protons from the cell to the lumen is a two-step process, with 
the first step requiring the activity of the $\mathrm{Na}^{+} / \mathrm{H}^{+}$exchanger NHX-2 (Nehrke, 2003). The second step occurs when the resulting proton gradient formed between the cytoplasm and the lumen presumably becomes too great for an electroneutral transporter such as NHX-2 to act against. Further pumping of protons from the cell requires the activity of a V-ATPase on the luminal membrane (Allman et al., 2009). Since the cell burns energy to pump protons back out of the intestinal cytoplasm into the lumen every $\sim 45 \mathrm{~s}$, this process is likely to be quite important, and loss of either $n h x-2$ or vha-6, a subunit of the intestinal apical V-ATPase, results in cellular acidosis and death (Nehrke, 2003; Allman et al., 2009).

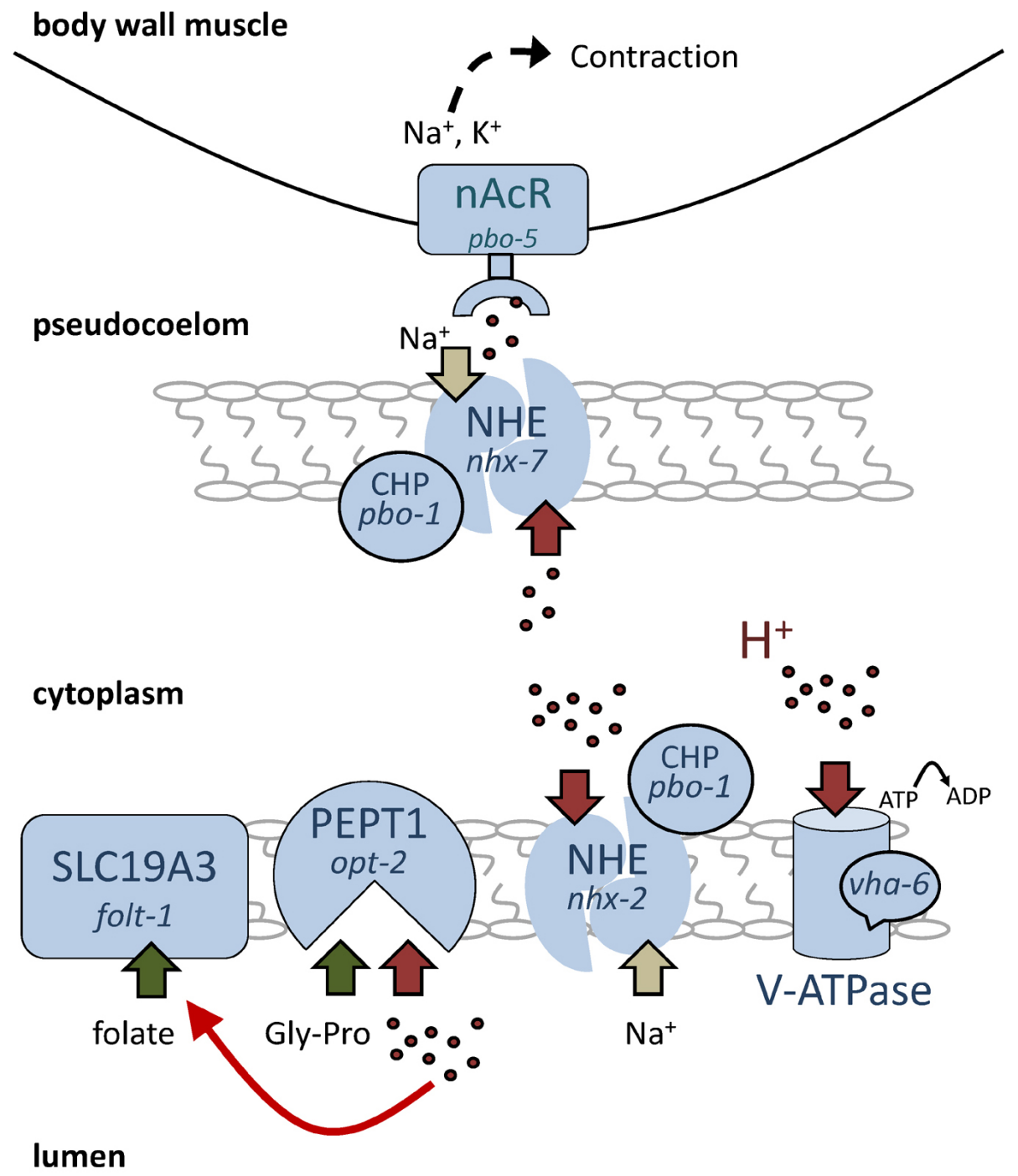

Figure 4. Protons at work: transport and signaling in the intestine. In this schematic diagram, protons are represented by red dots and their transport by red block arrows. Sodium transport is shown as tan arrows. Transport of nutrients such as dipeptide Gly-Pro or folate is represented by green arrows. At rest, a large $\mathrm{pH}$ gradient exists in the intestine, with the lumen $\mathrm{pH} 4$ « cytoplasm $\mathrm{pH} 7.5 \approx$ pseudocoelom $\mathrm{pH} 7.5$. During defecation, the lumen becomes more alkaline and the cytoplasm and pseudocoelom more acidic. Panel 3B represents the timing and magnitude of the changes in pH that occur during defecation relative to $\mathrm{Ca}^{2+}$ and the execution of the posterior body wall muscle contraction pBoc. Protons are a substrate for transport by sodium proton exchangers and V-ATPases, which maintain $\mathrm{pH}$ homeostasis, and for proton-dipeptide symporters, which take up dipeptide nutrients. Protons also regulate other transporters, such as SLC19A3, which is perhaps representative of a general role in nutrient uptake. On the basolateral side of the cell, activation of proton receptors allows adjacent cells to communicate and may represent a novel role for NHEs in this process. $\mathrm{Na}^{+} / \mathrm{H}^{+}$exchanger $(\mathrm{NHE})$, calcineurin homologous protein (CHP), nicotinic acetylcholine receptor (nAcR), proton-dipeptide co-transporter (PEPT1), adenosine triphosphate (ATP), vacuolar-type ATPase (V-ATPase), Solute Carrier Family 19, Member 3 (SLC19A3), glycine-proline dipeptide (Gly-Pro).

\subsection{Proton gradients and nutrient uptake}

Although one might predict that overt cellular acidosis is sufficient to cause death, one alternative, which is not mutually exclusive, is that it is instead the luminal protons or the proton gradient between the lumen and the cytoplasm that is essential for viability. Why would gut $\mathrm{pH}$ be important for viability? One possibility is suggested by the fact that many nutrient transporters require protons for their activity. There are a variety of nutrient 
transporters in worms whose activity has been shown to be coupled to proton co-transport or to be activated by protons (Fei et al., 1998; Xiao et al., 2001; Nehrke, 2003; Meissner et al., 2004). For example, the worm transporters NHX-2 and OPT-2 together facilitate proton-coupled dipeptide uptake (Nehrke, 2003; Meissner et al., 2004). This functional coupling is conserved in mammals, where $\mathrm{Na}^{+} / \mathrm{H}^{+}$exchanger isoform 3 drives oligopeptide absorption in the gut via the $\mathrm{H}^{+}$-dipeptide symporter PEPT1 (Kennedy et al., 2002; Thwaites et al., 2007). Subsequent dipeptide availability feeds into a variety of signaling pathways that recognize and respond to metabolic changes (for review see Spanier, 2013). Similarly, the folate transporter FOLT-1 in C. elegans is acid activated (Balamurugan et al., 2007) and folt-1 knockouts are sterile (Austin et al., 2010). Other possible targets that might be sensitive to luminal pH include SLC36 family members of proton-coupled amino acid transporters (Thwaites et al., 2011).

Consistent with this idea, a reduction in either $n h x-2$ or vha- 6 expression or activity is associated with reduced fat stores and increased lifespan (Nehrke, 2003; Allman et al., 2009; Wagner et al., 2011). This is consistent with the idea that nutrient deprivation extends lifespan but that too much deprivation-such as might occur in the loss-of-function $n h x-2$ and vha- 6 mutants-leads to starvation. The observation that $n h x$ - 2 lifespan extension is dependent upon PHA-4/FOXA (K. Nehrke, unpublished observations), a transcription factor that mediates the effects of caloric restriction on lifespan (Panowski et al., 2007), is consistent with this interpretation. One might therefore argue that the main purpose of defecation in worms is not to expulse the contents of the intestinal lumen, since mutant worms that lack expulsion (aex or exp mutants) are constipated but generally viable, but instead to conserve protons. By moving protons from the lumen into the cytoplasm, they are protected from being expulsed during defection, and in fact the $\mathrm{pH}$ of the expulsed waste is estimated to be $\approx 6$ (Pfeiffer et al., 2008). Subsequently pumping the protons back into the lumen is likely necessary for viability, based upon their role in nutrient uptake.

Recent research into folate metabolism illustrates how integrative physiological research in C. elegans can inform new approaches in higher level eukaryotes. Folate is normally synthesized by bacteria and absorbed in the worm gut through proton-dependent processes. Not surprisingly, a reduction in bacterial folate synthesis can extend worm lifespan (Virk et al., 2012). A similar extension of lifespan occurs from metformin (Onken et al., 2010), a commonly used drug prescribed to treat type 2 diabetes and metabolic syndrome. The mechanism leading to changes in lifespan of worms treated with metformin has been shown to involve altered microbial folate and methionine metabolism (Cabreiro et al., 2013). In mammals, the intestinal microbiome has also been shown to influence host metabolism, including development of metabolic disease. For example, groundbreaking new work has demonstrated "diet by microbiota" effects of gut microbes from human twins discordant for obesity following transplant into the intestines of germ-free mice (Ridaura et al., 2013). Perhaps there is an underlying change in folate metabolism in the twin's gut bacteria that transfers to the mice?

Indeed, beyond its direct relevance to nutrient uptake, the $\mathrm{pH}$ of the intestinal lumen is also likely to influence the rate at which bacteria proliferate therein (Portal-Celhay et al., 2012). Since worms encounter a variety of bacteria that end up in their gut, some of which are pathogenic, and the growth rate of discrete bacterial strains exhibit differing $\mathrm{pH}$ sensitivity, luminal proton homeostasis likely feeds into microbial pathogenesis and resulting innate immune responses. Interestingly, the immunologic defenses that mediate pathogen resistance have been shown to involve reactive oxygen species (ROS) formation and oxidative stress signaling (Chavez et al., 2007). ROS itself has been shown to cause cellular acidification in worms (Johnson et al., 2010) and can regulate the activity of the acid-base transporters such as $\mathrm{Na}^{+} / \mathrm{H}^{+}$exchangers found in the gut (Johnson et al., 2012). Ultimately, we come to the conclusion that $\mathrm{pH}$ is a dependent variable whose regulation both affects and is affected by multiple physiologic outputs. A truly integrative understanding of $\mathrm{pH}$ will take into account its effects on both host and microbiome.

\subsection{Proton signaling}

Historically, $\mathrm{pH}$ has been thought to provide "a metabolic context within and through which the actions of other effectors are integrated" (Busa et al., 1984). Similarly, the pathway through which information is transmitted between cells has typically been thought to involve neurotransmitter or peptide hormone secretion and receptor binding. Protons are considered as a modulator of ion channel function, if they are considered at all. However, this viewpoint was recently challenged by the finding that the $\mathrm{Na}^{+} / \mathrm{H}^{+}$exchanger $\mathrm{PBO}-4$ (also known as NHX-7), which resides on the basolateral membrane of the posterior intestinal cells, could extrude protons during defecation (Beg et al., 2008; Pfeiffer et al., 2008) and that protons could act as a ligand for the Cys-loop nicotinic type receptors PBO-5 and PBO-6 on the membrane of adjacent posterior body wall muscles (Beg et al., 2008). Uncaging protons with light induced pBoc in a PBO-5/6 dependent manner, confirming that proton modification of PBO-5/6 was both necessary and sufficient for signaling (Beg et al., 2008). 
This novel finding suggested that protons might act as signaling molecules in other contexts, as well. There are certainly scenarios where this type of signaling mechanism could be envisioned to be useful. For example, the balance between respiration and glycolysis can alter the rate of proton production in the cell, and cells could use proton signaling to communicate their metabolic status to their neighbors. In addition, because protons are capable of "hopping" across water molecules, this new mode of signaling could potentially transmit information between cells faster than the rate of diffusion, modifying how other, slower information is interpreted.

Interestingly, proton signaling appears to be discrete from transport mechanisms that regulate $\mathrm{pH}$ homeostasis. PBO-4 dependent proton extrusion across the basolateral membrane occurs acutely and transiently, and its loss does not appear to influence the rate at which $\mathrm{pH}$ homeostasis is recovered following defecation (Pfeiffer et al., 2008). In fact, PBO-4 is regulated by $\mathrm{Ca}^{2+}$ signaling through multiple parallel mechanisms such that its robust activity is restricted to a short period (Allman et al., 2013). This ensures that most of the protons that enter the cytoplasm during defecation will be shuttled back to the lumen, both reestablishing the proton gradient and reducing the possibility of metabolic acidosis occurring through excess proton secretion into the pseudocoelom. The activity of PBO-4 has also been shown to require PBO-1, a calcineurin homolog protein (CHP) ortholog and putative b-subunit that regulates $\mathrm{Na}^{+} / \mathrm{H}^{+}$exchangers in both worms and mammals (Peters et al., 2007). CHP loss of function is not tolerated in mammals, but the pbo- 1 deletion mutant is viable and should provide a valuable resource to decipher its mechanism of action in regulating $\mathrm{Na}^{+} / \mathrm{H}^{+}$exchangers in worms.

It appears likely that proton signaling is conserved in mammals, as the relevant proteins exist. Protons can be sensed by a class of G-protein coupled receptors including G2A, GPR4, OGR1, and TDAG8, with functions ranging from inflammation to renal acid secretion to osteoclastogenesis (Tomura et al., 2005), as well as by Acid Sensing Ion Channels (ASICs) involved in nociception (Deval et al., 2010). The nine member mammalian $\mathrm{Na}^{+} / \mathrm{H}^{+}$exchanger (NHE) gene family is widely expressed and one or more of these transporters may play an analogous function to PBO-4 in signaling. However, to date observations of $\mathrm{Na}^{+} / \mathrm{H}^{+}$exchanger-dependent proton extrusion as a signaling mechanism have been restricted to worms.

\section{Osmotic balance: the art of adaptation}

It's impossible to consider salt, or in this case ion transport, without also considering water. Systemic fluid and electrolyte balance is exquisitely maintained in all organisms. In general, this balance is regulated by the vectorial transport of ions (i.e. in one direction). Water is osmotically obliged to follow transported ions, often through aquaporins or water channels. As such, the transport of osmotically-active substances across the cell membrane can result in constant challenges to volume homeostasis.

Regulatory volume increase or decrease is a short-term mechanism by which electrolyte transport is triggered in response to cell shrinkage or swelling, as may occur during physiologic processes like fluid secretion or alternatively in response to environmental challenges such as exposure to high salt concentrations. Plasma membrane electrolyte transport processes are a major factor influencing the cell's and organism's ability to compensate appropriately to shrinkage or swelling through regulatory volume changes.

Worms have several tissues that contribute significantly to regulating whole-organism osmotic balance. The intestine and hypodermis appear to be particularly important in short term responses to changes in environmental salinity, and signaling pathways that contribute to systemic osmotic homeostasis following acute hypertonic challenge operate in these tissues (Choe et al., 2007). The excretory cell functions in long term osmoregulation and water balance, as laser ablation of this cell results in the worms bloating and dying within 24 hours. The regulated movement of salts in response to osmotic challenge occurs through a variety of transport mechanisms. Although the identity of the transporters themselves is of great interest, major contributions of work in the C. elegans model have involved defining upstream regulators of ion transport, specific targets of regulation, and downstream effectors of salt stress.

A particularly apt example of this is regulatory cascades that mediate $\mathrm{Cl}^{-}$transport. In mammals, loss-of-function mutations in the thiazide-sensitive $\mathrm{Na}^{+}-\mathrm{Cl}^{-}$cotransporter (NCC) cause phenotypes including hypertension, hyperkalemia, hyperchloremia, and metabolic acidosis, all of which depend upon the movement of chloride anion in the distal nephron (Simon et al., 1996). Hence, the regulation of NCC is critically important to human health. Shortly thereafter, human disease-causing mutants were identified in With-No-Lysine (K) Kinases WNK1 and WNK4 (Wilson et al., 2001) whose pseudohypoaldosteronism phenotypes, a diverse group of electrolyte metabolism disorders, mirrored the phenotypes resulting from loss of NCC. Over the past several years, the WNK 
signaling pathway has emerged as having a conserved role in regulating electrolyte transport. WNK kinases act upstream of Ste20-like proline/alanine-rich kinase (SPAK) and oxidative stress-responsive 1 (OSR1) kinases and in opposition to protein phosphatase 1 and calcineurin to regulate a variety of plasma membrane electrolyte transport proteins (for review see Kahle et al., 2010; Park et al., 2012).

The presence of single nematode gene representatives from the WNK subfamily (WNK-1) and the GCK VI subfamily of STE-20 protein kinases to which OSR1 and SPAK belong (GCK-3), has facilitated genetic approaches to determine their function (Strange et al., 2006). As regards cell volume regulation, tissue-specific RNAi suggests that GCK-3 and WNK-1 function in a common pathway in the hypodermis and intestine to regulate volume recovery following hypertonic shrinkage (Choe et al., 2007). The particular effectors for this process have not yet been identified, but are presumably transporters involved in maintaining systemic fluid and electrolyte balance.

GCK-3 was originally identified through an unbiased screen of proteins that interact with the cytosolic C-terminus of CLH-3b (Denton et al., 2005). CLH-3 is one of six ClC type $\mathrm{Cl}^{-}$channel family members in worms (Schriever et al., 1999; Nehrke et al., 2000) and CLH-3b is produced from a splice variant that is expressed in oocytes and activated during meiotic maturation (Rutledge et al., 2001). Meiotic maturation involves an enormous increase in oocyte volume. Recombinant CLH-3 activity can be triggered by volume changes but the native protein does not appear to be required for normal oocyte volume homeostasis during maturation. Instead, it plays a role in regulating sheath cell contractions through an as-of-yet incompletely understood cell non-autonomous mechanism (Rutledge et al., 2001). The net effect of CLH-3b activity is to synchronize oocyte cell cycle progression with ovulation and fertilization. The CLH-3b channel is normally kept inactive by GCK-3 dependent phosphorylation of S272 and S274, just downstream of its binding site (Denton et al., 2005; Falin et al., 2009). Activation of CLH-3b is a result of dephosphorylation by the type 1 phosphatases GSP-1 and GSP-2 (Rutledge et al., 2002). Extensive structure-function mutagenesis has provided striking insights into how alternative splicing regulates channel activity and how environmental information is transduced by phospho-regulation of its conformation and functional properties (Denton et al., 2004; He et al., 2006; Miyazaki et al., 2012; Miyazaki et al., 2012).

Surprisingly, despite the well documented involvement of WNK kinases in regulating mammalian transporters and its involvement upstream of GCK-3 in whole animal osmoregulation in C. elegans (Choe et al., 2007), CLH-3b regulation by GCK-3 during meiotic maturation does not involve WNK-1 (Falin et al., 2011). Instead, the ERK kinase MPK-1 and its upstream regulator MEK-2, which are known to play important roles in the meiotic cell cycle in $C$. elegans (Church et al., 1995; Leacock et al., 2006) appear to activate GCK-3, keeping CLH-3b basally repressed (Falin et al., 2011) (Figure 2). Hence, studies in worms have identified a new target transporter regulated by the OSR1/SPAK ortholog GCK-3 and a new kinase that participates in the regulatory cascade. Because of the clear role of WNK kinase in human health, it will be important to determine whether ERK kinases partner with GCK VI kinases in osmoregulatory cascades in mammals, as well.

In addition to its role in meiotic maturation, $g c k-3$ is widely expressed in worms and regulates several other developmental processes (Kupinski et al., 2010). Among these is tubular extension of the excretory canals. The worm excretory cell functions like a kidney, with canals that run along either side of the body being formed by seamless tubule extension from a single cell. Mutants in either $g c k-3$ or $w n k-1$ result in an excretory cell (exc mutant) phenotype (Hisamoto et al., 2008). In these mutants, the apical excretory membrane fails to extend as a result of a defect in triggering peri-apical vesicles to fuse with the lumen (Kolotuev et al., 2013). Since lumen expansion can occur in response to osmotic changes and involves the water channel AQP-8 (Khan et al., 2013) and the volume-sensitive $\mathrm{Cl}^{-}$channel CLH-3 (Hisamoto et al., 2008), a role for hydrodynamic force is suggested in the developmental process of tubule extension itself.

In contrast to short-term regulation of volume homeostasis through osmo-sensitive phosphorylation of electrolyte transporters, long-term approaches to surviving "salt stress" involve the induction of signaling pathways that prevent cell damage. An elegant review on this topic was published recently (Choe, 2013). Briefly, C elegans accumulate the organic osmolyte glycerol upon hypertonic exposure as a means of adaptation; this is reflected by transcriptional induction of GAPDH, the rate limiting enzyme in the production of glycerol (Lamitina et al., 2004). Reverse genetic screens, either using a $g p d h-1::$ GFP transcriptional reporter or scoring directly for hypertonicity resistance, identified proteostasis genes including components of the ubiquitin-proteasome system, endosomal sorting complexes, and lysosomes (Lamitina et al., 2006; Choe et al., 2008). In fact hypertonic stress causes rapid and widespread protein damage as measured by hypertonicity-induced aggregation of polyglutamine-YFP (Q35::YFP) (Burkewitz et al., 2011). The novel finding that inhibiting translation activates gpdh-1 gene transcription via WNK-1 and GCK-3 (Lee et al., 2012) delivers a direct link between acute and chronic adaptive mechanisms. 


\section{Conclusion}

The ease of experimental approaches and the wide repertoire of conserved membrane transport proteins contribute to $C$. elegans value as a genetic model for integrative physiological research. For example, the continuing evolution of fluorescent biosensors will allow sophisticated approaches to simultaneously image dynamic changes in organelle and cytoplasmic electrolyte concentrations. Combined with optogenetic approaches (Shipley et al., 2014) to manipulate $\mathrm{Ca}^{2+}$ or other second messengers, these reagents are likely to facilitate new avenues toward understanding how membrane ion flux influences systems biology. Exciting questions such as whether mitochondrial $\mathrm{Ca}^{2+}$ buffering integrates oscillatory $\mathrm{Ca}^{2+}$ signaling with metabolism or how cell stress/aging influences membrane transport are ready to be answered using existing molecular and genetic resources. New technologies for precisely manipulating the worm genome (Dickinson et al., 2013; Friedland et al., 2013) will facilitate in vivo structure-function analysis of transport proteins. New reagents such as genetically-caged $\mathrm{Ca}^{2+}$ (Fukuda et al., 2014) and genetically-encoded photosensitizers that use light to exert spatial and temporal control over ROS production (Wojtovich et al., 2014) will allow new approaches to be developed. Clearly, the field is expanding rapidly and the contributions of work in this area are certain to advance our understanding of mechanism, regulation, and functional output of electrolyte transporters.

\section{Acknowledgements}

Dr. Nehrke acknowledges support by USPHS NS064945 and GM087483 and NSF IOS 1352836. Thanks are due to Dr. Paul Brookes, Dr. Andrew Wojtovich and Dr. Andrew Samuelson for critical reading and suggestions.

\section{References}

Akerboom, J., Carreras Calderon, N., Tian, L., Wabnig, S., Prigge, M., Tolo, J., Gordus, A., Orger, M.B., Severi, K.E., Macklin, J.J., et al. (2013). Genetically encoded calcium indicators for multi-color neural activity imaging and combination with optogenetics. Front. Mol. Neurosci. 6, 2.Abstract Article

Allman, E., Johnson, D., and Nehrke, K. (2009). Loss of the apical V-ATPase a-subunit VHA-6 prevents acidification of the intestinal lumen during a rhythmic behavior in C. elegans. Am. J. Physiol. Cell Physiol. 297, C1071-1081.Abstract Article

Allman, E., Waters, K., Ackroyd, S., and Nehrke, K. (2013). Analysis of $\mathrm{Ca}^{2+}$ signaling motifs that regulate proton signaling through the $\mathrm{Na}^{+} / \mathrm{H}^{+}$exchanger NHX-7 during a rhythmic behavior in Caenorhabditis elegans. J. Biol. Chem. 288, 5886-5895.Abstract Article

Austin, M.U., Liau, W.S., Balamurugan, K., Ashokkumar, B., Said, H.M., and LaMunyon, C.W. (2010). Knockout of the folate transporter folt-1 causes germline and somatic defects in C. elegans. BMC Dev. Biol. 10, 46.Abstract Article

Balamurugan, K., Ashokkumar, B., Moussaif, M., Sze, J.Y., and Said, H.M. (2007). Cloning and functional characterization of a folate transporter from the nematode Caenorhabditis elegans. Am. J. Physiol. Cell Physiol. 293, C670-681.Abstract Article

Beg, A.A., Ernstrom, G.G., Nix, P., Davis, M.W., and Jorgensen, E.M. (2008). Protons act as a transmitter for muscle contraction in C. elegans. Cell 132, 149-160.Abstract Article

Bianchi, L. and Driscoll, M. Culture of embryonic C. elegans cells for electrophysiological and pharmacological analyses (September 30, 2006), WormBook, ed. The C. elegans Research Community, WormBook, doi/10.1895/wormbook.1.122.1, http://www.wormbook.org.Article

Branicky, R., and Hekimi, S. (2006). What keeps C. elegans regular: the genetics of defecation. Trends Genet. 22, 571-579.Abstract Article

Bretscher, A.J., Kodama-Namba, E., Busch, K.E., Murphy, R.J., Soltesz, Z., Laurent, P., and de Bono, M. (2011). Temperature, oxygen, and salt-sensing neurons in $C$. elegans are carbon dioxide sensors that control avoidance behavior. Neuron 69, 1099-1113.Abstract Article 
Bui, Y.K., and Sternberg, P.W. (2002). Caenorhabditis elegans inositol 5-phosphatase homolog negatively regulates inositol 1,4,5-triphosphate signaling in ovulation. Mol. Biol. Cell 13, 1641-1651.Abstract Article

Burkewitz, K., Choe, K., and Strange, K. (2011). Hypertonic stress induces rapid and widespread protein damage in C. elegans. Am. J. Physiol. Cell Physiol. 301, C566-576.Abstract Article

Busa, W.B., and Nuccitelli, R. (1984). Metabolic regulation via intracellular pH. Am. J. Physiol. 246, R409-438.Abstract

Cabreiro, F., Au, C., Leung, K.Y., Vergara-Irigaray, N., Cocheme, H.M., Noori, T., Weinkove, D., Schuster, E., Greene, N.D., and Gems, D. (2013). Metformin retards aging in C. elegans by altering microbial folate and methionine metabolism. Cell 153, 228-239.Abstract Article

Chavez, V., Mohri-Shiomi, A., Maadani, A., Vega, L.A., and Garsin, D.A. (2007). Oxidative stress enzymes are required for DAF-16-mediated immunity due to generation of reactive oxygen species by Caenorhabditis elegans. Genetics 176, 1567-1577.Abstract Article

Chen, H.C., Su, L.T., Gonzalez-Pagan, O., Overton, J.D., and Runnels, L.W. (2012). A key role for $\mathrm{Mg}^{2+}$ in TRPM7's control of ROS levels during cell stress. Biochem. J. 445, 441-448.Abstract Article

Cheon, B., Lee, H.C., Wakai, T., and Fissore, R.A. (2013). $\mathrm{Ca}^{2+}$ influx and the store-operated $\mathrm{Ca}^{2+}$ entry pathway undergo regulation during mouse oocyte maturation. Mol. Biol. Cell 24, 1396-1410.Abstract Article

Choe, K.P. (2013). Physiological and molecular mechanisms of salt and water homeostasis in the nematode Caenorhabditis elegans. Am. J. Physiol. Regul. Integr. Comp. Physiol. 305, R175-186.Abstract Article

Choe, K.P., and Strange, K. (2007). Evolutionarily conserved WNK and Ste20 kinases are essential for acute volume recovery and survival after hypertonic shrinkage in Caenorhabditis elegans. Am. J. Physiol. Cell Physiol. 293, C915-927.Abstract Article

Choe, K.P., and Strange, K. (2008). Genome-wide RNAi screen and in vivo protein aggregation reporters identify degradation of damaged proteins as an essential hypertonic stress response. Am. J. Physiol. Cell Physiol. 295, C1488-1498.Abstract Article

Christensen, M., Estevez, A., Yin, X., Fox, R., Morrison, R., McDonnell, M., Gleason, C., Miller, D.M., 3rd and Strange, K. (2002). A primary culture system for functional analysis of C. elegans neurons and muscle cells. Neuron 33, 503-514.Abstract Article

Church, D.L., Guan, K.L., and Lambie, E.J. (1995). Three genes of the MAP kinase cascade, mek-2, mpk-1/sur-1 and let-60 ras, are required for meiotic cell cycle progression in Caenorhabditis elegans. Development 121, 2525-2535.Abstract

Clandinin, T.R., DeModena, J.A., and Sternberg, P.W. (1998). Inositol trisphosphate mediates a RAS-independent response to LET-23 receptor tyrosine kinase activation in C. elegans. Cell 92, 523-533.Abstract Article

Coburn, C., Allman, E., Mahanti, P., Benedetto, A., Cabreiro, F., Pincus, Z., Matthijssens, F., Araiz, C., Mandel, A., Vlachos, M., et al. (2013). Anthranilate fluorescence marks a calcium-propagated necrotic wave that promotes organismal death in C. elegans. PLoS Biol. 11, e1001613.Abstract Article

Corrigan, C., Subramanian, R., and Miller, M.A. (2005). Eph and NMDA receptors control $\mathrm{Ca}^{2+}$ /calmodulin-dependent protein kinase II activation during C. elegans oocyte meiotic maturation. Development 132, 5225-5237.Abstract Article

Dal Santo, P., Logan, M.A., Chisholm, A.D., and Jorgensen, E.M. (1999). The inositol trisphosphate receptor regulates a 50-second behavioral rhythm in C. elegans. Cell 98, 757-767.Abstract Article

Denton, J., Nehrke, K., Rutledge, E., Morrison, R., and Strange, K. (2004). Alternative splicing of N- and C-termini of a C. elegans $\mathrm{ClC}$ channel alters gating and sensitivity to external $\mathrm{Cl}^{-}$and $\mathrm{H}^{+}$. J. Physiol. 555, 97-114.Abstract Article 
Denton, J., Nehrke, K., Yin, X., Morrison, R., and Strange, K. (2005). GCK-3, a newly identified Ste20 kinase, binds to and regulates the activity of a cell cycle-dependent $\mathrm{ClC}$ anion channel. J. Gen. Physiol. 125, 113-125.Abstract Article

Deval, E., Gasull, X., Noel, J., Salinas, M., Baron, A., Diochot, S., and Lingueglia, E. (2010). Acid-sensing ion channels (ASICs): pharmacology and implication in pain. Pharmacol. Ther. 128, 549-558.Abstract Article

Dickinson, D.J., Ward, J.D., Reiner, D.J., and Goldstein, B. (2013). Engineering the Caenorhabditis elegans genome using Cas9-triggered homologous recombination. Nat. Methods 10, 1028-1034.Abstract Article

Espelt, M.V., Estevez, A.Y., Yin, X., and Strange, K. (2005). Oscillatory $\mathrm{Ca}^{2+}$ signaling in the isolated Caenorhabditis elegans intestine: role of the inositol-1,4,5-trisphosphate receptor and phospholipases $\mathrm{C} \beta$ and $\gamma$. J. Gen. Physiol. 126, 379-392.Abstract Article

Estevez, A.Y., Roberts, R.K., and Strange, K. (2003). Identification of store-independent and store-operated Ca ${ }^{2+}$ conductances in Caenorhabditis elegans intestinal epithelial cells. J. Gen. Physiol. 122, 207-223.Abstract Article

Estevez, A.Y., and Strange, K. (2005). Calcium feedback mechanisms regulate oscillatory activity of a TRP-like $\mathrm{Ca}^{2+}$ conductance in C. elegans intestinal cells. J. Physiol. 567, 239-251.Abstract Article

Falin, R.A., Miyazaki, H., and Strange, K. (2011). C. elegans STK39/SPAK ortholog-mediated inhibition of ClC anion channel activity is regulated by WNK-independent ERK kinase signaling. Am. J. Physiol. Cell Physiol. 300, C624-635.Abstract Article

Falin, R.A., Morrison, R., Ham, A.J., and Strange, K. (2009). Identification of regulatory phosphorylation sites in a cell volume- and Ste20 kinase-dependent ClC anion channel. J. Gen. Physiol. 133, 29-42.Abstract Article

Fasseas, M.K., Tsikou, D., Flemetakis, E., and Katinakis, P. (2011). Molecular and biochemical analysis of the alpha class carbonic anhydrases in Caenorhabditis elegans. Mol. Biol. Rep. 38, 1777-1785.Abstract Article

Fei, Y.J., Fujita, T., Lapp, D.F., Ganapathy, V., and Leibach, F.H. (1998). Two oligopeptide transporters from Caenorhabditis elegans: molecular cloning and functional expression. Biochem. J. 332 ( Pt 2), 565-572.Abstract

Friedland, A.E., Tzur, Y.B., Esvelt, K.M., Colaiacovo, M.P., Church, G.M., and Calarco, J.A. (2013). Heritable genome editing in C. elegans via a CRISPR-Cas9 system. Nat. Methods 10, 741-743.Abstract Article

Fukuda, N., Matsuda, T., and Nagai, T. (2014). Optical control of the $\mathrm{Ca}^{2+}$ concentration in a live specimen with a genetically encoded $\mathrm{Ca}^{2+}$-releasing molecular tool. ACS Chem. Biol. 9, 1197-1203.Abstract Article

Goodman, M.B., Hall, D.H., Avery, L., and Lockery, S.R. (1998). Active currents regulate sensitivity and dynamic range in C. elegans neurons. Neuron 20, 763-772.Abstract Article

Goodman, M.B., Lindsay, T.H., Lockery, S.R., and Richmond, J.E. (2012). Electrophysiological methods for Caenorhabditis elegans neurobiology. Methods Cell Biol. 107, 409-436.Abstract Article

Govindan, J.A., Cheng, H., Harris, J.E., and Greenstein, D. (2006). G $\alpha_{\mathrm{o} / \mathrm{i}}$ and $\mathrm{G} \alpha_{\mathrm{s}}$ signaling function in parallel with the MSP/Eph receptor to control meiotic diapause in C. elegans. Curr. Biol. 16, 1257-1268.Abstract Article

Greenstein, D. Control of oocyte meiotic maturation and fertilization (December 28, 2005), WormBook, ed. The C. elegans Research Community, WormBook, doi/10.1895/wormbook.1.53.1, http://www.wormbook.org.Article

He, L., Denton, J., Nehrke, K., and Strange, K. (2006). Carboxy terminus splice variation alters ClC channel gating and extracellular cysteine reactivity. Biophys. J. 90, 3570-3581.Abstract Article

Hisamoto, N., Moriguchi, T., Urushiyama, S., Mitani, S., Shibuya, H., and Matsumoto, K. (2008). Caenorhabditis elegans WNK-STE20 pathway regulates tube formation by modulating ClC channel activity. EMBO Rep 9, 70-75.Abstract Article 
Huang, C.L. (2007). Complex roles of PIP2 in the regulation of ion channels and transporters. Am. J. Physiol. Renal Physiol. 293, F1761-1765.Abstract Article

Johnson, D., Allman, E., and Nehrke, K. (2012). Regulation of acid-base transporters by reactive oxygen species following mitochondrial fragmentation. Am. J. Physiol. Cell Physiol. 302, C1045-1054.Abstract Article

Johnson, D., and Nehrke, K. (2010). Mitochondrial fragmentation leads to intracellular acidification in Caenorhabditis elegans and mammalian cells. Mol. Biol. Cell 21, 2191-2201.Abstract Article

Kahle, K.T., Rinehart, J., and Lifton, R.P. (2010). Phosphoregulation of the Na-K-2Cl and K-Cl cotransporters by the WNK kinases. Biochim. Biophys. Acta 1802, 1150-1158.Abstract Article

Kariya, K., Bui, Y.K., Gao, X., Sternberg, P.W., and Kataoka, T. (2004). Phospholipase Ce regulates ovulation in Caenorhabditis elegans. Dev. Biol. 274, 201-210.Abstract Article

Kemp, B.J., Allman, E., Immerman, L., Mohnen, M., Peters, M.A., Nehrke, K., and Abbott, A.L. (2012). miR-786 regulation of a fatty-acid elongase contributes to rhythmic calcium-wave initiation in C. elegans. Curr. Biol. 22, 2213-2220.Abstract Article

Kennedy, D.J., Leibach, F.H., Ganapathy, V., and Thwaites, D.T. (2002). Optimal absorptive transport of the dipeptide glycylsarcosine is dependent on functional $\mathrm{Na}^{+} / \mathrm{H}^{+}$exchange activity. Pflugers Arch. 445, 139-146.Abstract Article

Khan, L.A., Zhang, H., Abraham, N., Sun, L., Fleming, J.T., Buechner, M., Hall, D.H., and Gobel, V. (2013). Intracellular lumen extension requires ERM-1-dependent apical membrane expansion and AQP-8-mediated flux. Nat. Cell Biol. 15, 143-156.Abstract Article

Kolotuev, I., Hyenne, V., Schwab, Y., Rodriguez, D., and Labouesse, M. (2013). A pathway for unicellular tube extension depending on the lymphatic vessel determinant Prox1 and on osmoregulation. Nat. Cell Biol. 15, 157-168.Abstract Article

Kovacevic, I., Orozco, J.M., and Cram, E.J. (2013). Filamin and phospholipase C- $\varepsilon$ are required for calcium signaling in the Caenorhabditis elegans spermatheca. PLoS Genet. 9, e1003510.Abstract Article

Kupinski, A.P., Muller-Reichert, T., and Eckmann, C.R. (2010). The Caenorhabditis elegans Ste20 kinase, GCK-3, is essential for postembryonic developmental timing and regulates meiotic chromosome segregation. Dev. Biol. 344, 758-771.Abstract Article

Kwan, C.S., Vazquez-Manrique, R.P., Ly, S., Goyal, K., and Baylis, H.A. (2008). TRPM channels are required for rhythmicity in the ultradian defecation rhythm of $C$. elegans. BMC Physiol. 8, 11.Abstract Article

Lamitina, S.T., Morrison, R., Moeckel, G.W., and Strange, K. (2004). Adaptation of the nematode Caenorhabditis elegans to extreme osmotic stress. Am. J. Physiol. Cell Physiol. 286, C785-791.Abstract Article

Lamitina, T., Huang, C.G., and Strange, K. (2006). Genome-wide RNAi screening identifies protein damage as a regulator of osmoprotective gene expression. Proc. Natl. Acad. Sci. U. S. A. 103, 12173-12178.Abstract Article

Leacock, S.W., and Reinke, V. (2006). Expression profiling of MAP kinase-mediated meiotic progression in Caenorhabditis elegans. PLoS Genet. 2, e174.Abstract Article

Lee, E.C., and Strange, K. (2012). GCN-2 dependent inhibition of protein synthesis activates osmosensitive gene transcription via WNK and Ste20 kinase signaling. Am. J. Physiol. Cell Physiol. 303, C1269-1277.Abstract Article

Liu, D.W., and Thomas, J.H. (1994). Regulation of a periodic motor program in C. elegans. J. Neurosci. 14, 1953-1962.Abstract

Lorin-Nebel, C., Xing, J., Yan, X., and Strange, K. (2007). CRAC channel activity in C. elegans is mediated by Orai1 and STIM1 homologues and is essential for ovulation and fertility. J. Physiol. 580, 67-85.Abstract Article 
Meissner, B., Boll, M., Daniel, H., and Baumeister, R. (2004). Deletion of the intestinal peptide transporter affects insulin and TOR signaling in Caenorhabditis elegans. J. Biol. Chem. 279, 36739-36745.Abstract Article

Miller, K.G., Emerson, M.D., and Rand, J.B. (1999). $\mathrm{G}_{\mathrm{o}} \alpha$ and diacylglycerol kinase negatively regulate the $\mathrm{G}_{\mathrm{q}} \alpha$ pathway in C. elegans. Neuron 24, 323-333.Abstract Article

Miller, M.A., Nguyen, V.Q., Lee, M.H., Kosinski, M., Schedl, T., Caprioli, R.M., and Greenstein, D. (2001). A sperm cytoskeletal protein that signals oocyte meiotic maturation and ovulation. Science 291, 2144-2147.Abstract Article

Miyazaki, H., and Strange, K. (2012). Differential regulation of a CLC anion channel by SPAK kinase ortholog-mediated multisite phosphorylation. Am. J. Physiol. Cell Physiol. 302, C1702-1712.Abstract Article

Miyazaki, H., Yamada, T., Parton, A., Morrison, R., Kim, S., Beth, A.H., and Strange, K. (2012). CLC anion channel regulatory phosphorylation and conserved signal transduction domains. Biophys. J. 103, 1706-1718.Abstract Article

Nehrke, K. (2003). A reduction in intestinal cell pHi due to loss of the Caenorhabditis elegans $\mathrm{Na}^{+} / \mathrm{H}^{+}$exchanger NHX-2 increases life span. J. Biol. Chem. 278, 44657-44666.Abstract Article

Nehrke, K., Begenisich, T., Pilato, J., and Melvin, J.E. (2000). Into ion channel and transporter function. Caenorhabditis elegans ClC-type chloride channels: novel variants and functional expression. Am. J. Physiol. Cell Physiol. 279, C2052-2066.Abstract

Nehrke, K., Denton, J., and Mowrey, W. (2008). Intestinal $\mathrm{Ca}^{2+}$ wave dynamics in freely moving C. elegans coordinate execution of a rhythmic motor program. Am. J. Physiol. Cell Physiol. 294, C333-344.Abstract Article

Nehrke, K., and Melvin, J.E. (2002). The NHX family of $\mathrm{Na}^{+}-\mathrm{H}^{+}$exchangers in Caenorhabditis elegans. J. Biol. Chem. 277, 29036-29044.Abstract Article

Norman, K.R., Fazzio, R.T., Mellem, J.E., Espelt, M.V., Strange, K., Beckerle, M.C., and Maricq, A.V. (2005). The Rho/Rac-family guanine nucleotide exchange factor VAV-1 regulates rhythmic behaviors in C. elegans. Cell 123, 119-132.Abstract Article

Onken, B., and Driscoll, M. (2010). Metformin induces a dietary restriction-like state and the oxidative stress response to extend $C$. elegans healthspan via AMPK, LKB1, and SKN-1. PLoS One 5, e8758.Abstract Article

Panowski, S.H., Wolff, S., Aguilaniu, H., Durieux, J., and Dillin, A. (2007). PHA-4/Foxa mediates diet-restriction-induced longevity of C. elegans. Nature 447, 550-555.Abstract Article

Park, S., Hong, J.H., Ohana, E., and Muallem, S. (2012). The WNK/SPAK and IRBIT/PP1 pathways in epithelial fluid and electrolyte transport. Physiology 27, 291-299.Abstract Article

Peters, M.A., Teramoto, T., White, J.Q., Iwasaki, K., and Jorgensen, E.M. (2007). A calcium wave mediated by gap junctions coordinates a rhythmic behavior in C. elegans. Curr. Biol. 17, 1601-1608.Abstract Article

Pfeiffer, J., Johnson, D., and Nehrke, K. (2008). Oscillatory transepithelial $\mathrm{H}^{+}$flux regulates a rhythmic behavior in C. elegans. Curr. Biol. 18, 297-302.Abstract Article

Portal-Celhay, C., Bradley, E.R., and Blaser, M.J. (2012). Control of intestinal bacterial proliferation in regulation of lifespan in Caenorhabditis elegans. BMC Microbiol. 12, 49.Abstract Article

Prakriya, M., Feske, S., Gwack, Y., Srikanth, S., Rao, A., and Hogan, P.G. (2006). Orai1 is an essential pore subunit of the CRAC channel. Nature 443, 230-233.Abstract Article

Quamme, G.A. (2008). Recent developments in intestinal magnesium absorption. Curr. Opin. Gastroenterol. 24, 230-235.Abstract Article 
Razzell, W., Evans, I.R., Martin, P., and Wood, W. (2013). Calcium flashes orchestrate the wound inflammatory response through DUOX activation and hydrogen peroxide release. Curr. Biol. 23, 424-429.Abstract Article

Richmond, J.E., and Jorgensen, E.M. (1999). One GABA and two acetylcholine receptors function at the C. elegans neuromuscular junction. Nat. Neurosci. 2, 791-797.Abstract Article

Ridaura, V.K., Faith, J.J., Rey, F.E., Cheng, J., Duncan, A.E., Kau, A.L., Griffin, N.W., Lombard, V., Henrissat, B., Bain, et al. (2013). Gut microbiota from twins discordant for obesity modulate metabolism in mice. Science 341, 1241214.Abstract Article

Roos, J., DiGregorio, P.J., Yeromin, A.V., Ohlsen, K., Lioudyno, M., Zhang, S., Safrina, O., Kozak, J.A., Wagner, S.L., Cahalan, M.D., Velicelebi, G., and Stauderman, K.A. (2005). STIM1, an essential and conserved component of store-operated $\mathrm{Ca}^{2+}$ channel function. J. Cell Biol. 169, 435-445.Abstract Article

Runnels, L.W. (2011). TRPM6 and TRPM7: A Mul-TRP-PLIK-cation of channel functions. Curr. Pharm. Biotechnol. 12, 42-53.Abstract Article

Rutledge, E., Bianchi, L., Christensen, M., Boehmer, C., Morrison, R., Broslat, A., Beld, A.M., George, A.L., Greenstein, D., and Strange, K. (2001). CLH-3, a ClC-2 anion channel ortholog activated during meiotic maturation in C. elegans oocytes. Curr. Biol. 11, 161-170.Abstract Article

Rutledge, E., Denton, J., and Strange, K. (2002). Cell cycle- and swelling-induced activation of a Caenorhabditis elegans $\mathrm{ClC}$ channel is mediated by CeGLC-7alpha/beta phosphatases. J. Cell Biol. 158, 435-444.Abstract Article

Schriever, A.M., Friedrich, T., Pusch, M., and Jentsch, T.J. (1999). CLC chloride channels in Caenorhabditis elegans. J. Biol. Chem. 274, 34238-34244.Abstract Article

Sherman, T., Chernova, M.N., Clark, J.S., Jiang, L., Alper, S.L., and Nehrke, K. (2005). The abts and sulp families of anion transporters from Caenorhabditis elegans. Am. J. Physiol. Cell Physiol. 289, C341-351.Abstract Article

Sherman, T.A., Rongali, S.C., Matthews, T.A., Pfeiffer, J., and Nehrke, K. (2012). Identification of a nuclear carbonic anhydrase in Caenorhabditis elegans. Biochim. Biophys. Acta 1823, 808-817.Abstract Article

Shipley, F.B., Clark, C.M., Alkema, M.J., and Leifer, A.M. (2014). Simultaneous optogenetic manipulation and calcium imaging in freely moving C. elegans. Front. Neural Circuits 8, 28.Abstract Article

Simon, D.B., Nelson-Williams, C., Bia, M.J., Ellison, D., Karet, F.E., Molina, A.M., Vaara, I., Iwata, F., Cushner, H.M., Koolen, M., et al. (1996). Gitelman's variant of Bartter's syndrome, inherited hypokalaemic alkalosis, is caused by mutations in the thiazide-sensitive Na-Cl cotransporter. Nat. Genet. 12, 24-30.Abstract Article

Singaravelu, G., and Singson, A. (2013). Calcium signaling surrounding fertilization in the nematode Caenorhabditis elegans. Cell Calcium 53, 2-9.Abstract Article

Spanier, B. (2013). Transcriptional and functional regulation of the intestinal peptide transporter PEPT1. J. Physiol. 529, 871-879.Abstract

Stawicki, T.M., Zhou, K., Yochem, J., Chen, L., and Jin, Y. (2011). TRPM channels modulate epileptic-like convulsions via systemic ion homeostasis. Curr. Biol. 21, 883-888.Abstract Article

Strange, K., Christensen, M., and Morrison, R. (2007). Primary culture of Caenorhabditis elegans developing embryo cells for electrophysiological, cell biological and molecular studies. Nat. Protoc. 2, 1003-1012.Abstract Article

Strange, K., Denton, J., and Nehrke, K. (2006). Ste20-type kinases: evolutionarily conserved regulators of ion transport and cell volume. Physiology 21, 61-68.Abstract Article

Strange, K., Yan, X., Lorin-Nebel, C., and Xing, J. (2007). Physiological roles of STIM1 and Orai1 homologs and CRAC channels in the genetic model organism Caenorhabditis elegans. Cell Calcium 42, 193-203.Abstract Article 
Sumoza-Toledo, A., and Penner, R. (2011). TRPM2: a multifunctional ion channel for calcium signalling. J. Physiol. 589, 1515-1525.Abstract Article

Suzuki, N., and Mittler, R. (2012). Reactive oxygen species-dependent wound responses in animals and plants. Free Radic. Biol. Med. 53, 2269-2276.Abstract Article

Teramoto, T., and Iwasaki, K. (2006). Intestinal calcium waves coordinate a behavioral motor program in $C$. elegans. Cell Calcium 40, 319-327.Abstract Article

Teramoto, T., Lambie, E.J., and Iwasaki, K. (2005). Differential regulation of TRPM channels governs electrolyte homeostasis in the C. elegans intestine. Cell Metab 1, 343-354.Abstract Article

Teramoto, T., Sternick, L.A., Kage-Nakadai, E., Sajjadi, S., Siembida, J., Mitani, S., Iwasaki, K., and Lambie, E.J. (2010). Magnesium excretion in C. elegans requires the activity of the GTL-2 TRPM channel. PLoS One 5, e9589.Abstract Article

Thomas, J.H. (1990). Genetic analysis of defecation in Caenorhabditis elegans. Genetics 124, 855-872.Abstract

Thwaites, D.T., and Anderson, C.M. (2007). $\mathrm{H}^{+}$-coupled nutrient, micronutrient and drug transporters in the mammalian small intestine. Exp. Physiol. 92, 603-619.Abstract Article

Thwaites, D.T., and Anderson, C.M. (2011). The SLC36 family of proton-coupled amino acid transporters and their potential role in drug transport. Br. J. Pharmacol. 164, 1802-1816.Abstract Article

Tomura, H., Mogi, C., Sato, K., and Okajima, F. (2005). Proton-sensing and lysolipid-sensitive G-protein-coupled receptors: a novel type of multi-functional receptors. Cell. Signal. 17, 1466-1476.Abstract Article

Virk, B., Correia, G., Dixon, D.P., Feyst, I., Jia, J., Oberleitner, N., Briggs, Z., Hodge, E., Edwards, R., Ward, J., et al. (2012). Excessive folate synthesis limits lifespan in the C. elegans: E. coli aging model. BMC Biol. 10, 67.Abstract Article

Wagner, J., Allman, E., Taylor, A., Ulmschneider, K., Kovanda, T., Ulmschneider, B., Nehrke, K., and Peters, M.A. (2011). A calcineurin homologous protein is required for sodium-proton exchange events in the C. elegans intestine. Am. J. Physiol. Cell Physiol. 301, C1389-1403.Abstract Article

Wang, H., Girskis, K., Janssen, T., Chan, J.P., Dasgupta, K., Knowles, J.A., Schoofs, L., and Sieburth, D. (2013). Neuropeptide secreted from a pacemaker activates neurons to control a rhythmic behavior. Curr. Biol. 23, 746-754.Abstract Article

Wilson, F.H., Disse-Nicodeme, S., Choate, K.A., Ishikawa, K., Nelson-Williams, C., Desitter, I., Gunel, M., Milford, D.V., Lipkin, G.W., Achard, J.M., et al. (2001). Human hypertension caused by mutations in WNK kinases. Science 293, 1107-1112.Abstract Article

Wojtovich, A.P., and Foster, T.H. (2014). Optogenetic control of ROS production. Redox Biol. 2, 368-376.Abstract Article

Xiao, G., Wang, J., Tangen, T., and Giacomini, K.M. (2001). A novel proton-dependent nucleoside transporter, CeCNT3, from Caenorhabditis elegans. Mol. Pharmacol. 59, 339-348.Abstract

Xing, J., and Strange, K. (2010). Phosphatidylinositol 4,5-bisphosphate and loss of PLC $\gamma$ activity inhibit TRPM channels required for oscillatory $\mathrm{Ca}^{2+}$ signaling. Am. J. Physiol. Cell Physiol. 298, C274-282.Abstract Article

Xing, J., Yan, X., Estevez, A., and Strange, K. (2008). Highly $\mathrm{Ca}^{2+}$-selective TRPM channels regulate IP3-dependent oscillatory $\mathrm{Ca}^{2+}$ signaling in the C. elegans intestine. J. Gen. Physiol. 131, 245-255.Abstract Article

$\mathrm{Xu}$, S., and Chisholm, A.D. (2011). A $\mathrm{G \alpha}_{\mathrm{q}}-\mathrm{Ca}^{2+}$ signaling pathway promotes actin-mediated epidermal wound closure in C. elegans. Curr. Biol. 21, 1960-1967.Abstract Article 
Yan, X., Xing, J., Lorin-Nebel, C., Estevez, A.Y., Nehrke, K., Lamitina, T., and Strange, K. (2006). Function of a STIM1 homologue in $C$. elegans: evidence that store-operated $\mathrm{Ca}^{2+}$ entry is not essential for oscillatory Ca signaling and ER Ca ${ }^{2+}$ homeostasis. J. Gen. Physiol. 128, 443-459.Abstract Article

Yeromin, A.V., Zhang, S.L., Jiang, W., Yu, Y., Safrina, O., and Cahalan, M.D. (2006). Molecular identification of the CRAC channel by altered ion selectivity in a mutant of Orai. Nature 443, 226-229.Abstract Article

Yin, X., Gower, N.J., Baylis, H.A., and Strange, K. (2004). Inositol 1,4,5-trisphosphate signaling regulates rhythmic contractile activity of myoepithelial sheath cells in Caenorhabditis elegans. Mol. Biol. Cell 15, 3938-3949.Abstract Article

Zhang, S.L., Yu, Y., Roos, J., Kozak, J.A., Deerinck, T.J., Ellisman, M.H., Stauderman, K.A., and Cahalan, M.D. (2005). STIM1 is a $\mathrm{Ca}^{2+}$ sensor that activates CRAC channels and migrates from the $\mathrm{Ca}^{2+}$ store to the plasma membrane. Nature 437, 902-905.Abstract Article

All WormBook content, except where otherwise noted, is licensed under a Creative SOMRERIGHISRESERVED Commons Attribution License. 